\title{
Cooling out after breaking up. Milieuunterschiede im Umgang mit dem Beziehungsscheitern
}

\author{
Judith Eckert
}

Angenommen: 21. September 2021 / Online publiziert: 11. Oktober 2021

(C) Der/die Autor(en) 2021

Zusammenfassung In seinem Aufsatz „On cooling the mark out. Some aspects of adaptation to failure" erwähnt Erving Goffman das Scheitern von Paarbeziehungen als Paradebeispiel für Cooling-out-Prozesse in informellen sozialen Settings. Daran anknüpfend fragt der vorliegende Beitrag zum einen, inwiefern das Cooling-outKonzept für die gegenwärtige familiensoziologische Theoriebildung fruchtbar gemacht werden kann. Indem es, so wird argumentiert, ein integratives und genuin soziologisches Instrumentarium zum Verständnis materieller und vor allem symbolischer Trennungsfolgen, die damit einhergehenden interpretativen Krisen sowie deren Bewältigung bietet, hat Goffmans Konzept gegenüber den bislang dominierenden psychologischen Konzeptualisierungen deutliche Vorzüge. Zum anderen wird danach gefragt, inwiefern die Analyse des Umgangs mit dem Scheitern von Paarbeziehungen zur Weiterentwicklung des Cooling-out-Konzepts beitragen kann. Auf der Grundlage qualitativer Interviews mit Getrennten wird gezeigt, dass es milieuspezifische Präferenzen bezüglich der ,abkühlenden“ Deutungsrahmen gibt, auf die die Akteure bei der Situations- und Selbstredefinition zurückgreifen. Während im individualisierten Milieu die (Re-)Konstruktion ,verborgener Wahrheiten“ in vertrauensvollen Gesprächen und die reflexive Arbeit am Selbst im Vordergrund steht, ist im traditionalen Milieu die Mobilisierung öffentlicher Fürsprache angesichts infrage stehender Geschlechterrollenperformanz von zentraler Bedeutung. Erweitert um Goffmans Rahmenanalyse und milieusoziologisch angewandt, lassen sich somit mithilfe des Cooling-out-Konzepts stark divergierende Deutungsmuster und Praxen in der Verarbeitung von Trennungen beschreiben.

Schlüsselwörter Cooling out · Rahmenanalyse · Trennung · Trennungsfolgen · Milieu 


\section{Cooling out after breaking up. Milieu differences in dealing with relationship failure}

Abstract In his article "On cooling the mark out. Some aspects of adaptation to failure", Goffman calls the failure of couples' relationships a prime example of cooling out processes in informal social settings. Against this background, the paper discusses how, on the one hand, theorization in the sociology of the family can benefit from the concept of cooling out. It is argued that Goffman's concept has considerable advantages over prevalent psychological approaches by offering an integrative and genuinely sociological tool fit for grasping the material and, above all, symbolic consequences of separation as well as the corresponding interpretative crises and coping attempts. On the other hand, the article asks how analysing breakups may serve the further development of the cooling out concept. Based on qualitative interviews with ex-partners, the article shows the existence of milieu-specific preference regarding the interpretive frameworks of "cooling out" that the actors resort to while trying to redefine the situation and their selves. While the (re)construction of "hidden truths" in intimate conversations and the work on one's self is paramount in the individualist milieu, members of the traditional milieu are rather looking for reassurance concerning their gender role performance by mobilizing public support. Complemented with Goffman's "frame analysis" and applied in a milieu-oriented perspective, the cooling out concept proves useful by allowing for the identification of starkly diverging interpretative patterns and practices in dealing with breakups.

Keywords Cooling out · Frame analysis · Breaking up · Consequences of separarations · Milieu

\section{Cooling out after breaking up: comment l'échec du couple est géré dans différents milieux sociaux}

Résumé Dans son article „On cooling the mark out. Some aspects of adaptation to failure" Goffman évoque l'échec des relations de couple comme un exemple typique des processus de cooling-out (refroidissement) dans des contextes sociaux informels. Dans le prolongement des travaux de Goffmann, le présent article se consacre à deux questions à partir d'entretiens qualitatifs avec des ex-conjoint.e.s. Il discute tout d'abord la question de savoir dans quelle mesure le concept de coolingout peut être mis à profit pour l'élaboration de théories en sociologie de la famille. L'argument présenté est que la pertinence de ce concept réside en ceci qu'il offre un cadre proprement sociologique permettant d'intégrer les différentes conséquences matérielles et surtout symboliques empiriquement constatables qui résultent de la séparation ou du divorce, les crises interprétatives qui s'ensuivent ainsi que la gestion de celles-ci. L'article s'interroge, inversement, sur la contribution que l'analyse de la gestion de l'échec des relations de couple peut apporter au développement du concept de cooling-out. À partir d'entretiens qualitatifs avec des personnes divorcées, il est notamment mis en évidence qu'il existe des préférences propres à chaque milieu quant au cadre d'interprétation „refroidissant“ auquel les acteurs recourent pour définir la situation et eux-mêmes. Dans le milieu individualiste, la (re)construction 
de „vérités cachées“ dans le cadre de discussions intimes et le travail réflexif sur soi sont au premier plan ; dans le milieu traditionnel, en revanche, la mobilisation de la parole publique face à la mise en question de la performance des rôles sexuels est cruciale. Enrichi de l'analyse des cadres de Goffman et employé dans la sociologie des milieux sociaux, le concept de cooling out permet ainsi de décrire des modèles d'interprétation et des pratiques largement divergents en matière de gestion des séparations.

Mots-clés Cooling-out · Analyse des cadres · Séparation · Conséquences de la séparation · Milieu social

\section{Einleitung}

Wie im Untertitel seines Cooling-out-Aufsatzes angedeutet, geht es Goffman (1952) bei der Frage nach dem Cooling-out um Arten und Weisen der ,,adaptation to failure“, also um den sozialen Umgang mit Verlust und Scheitern. Ausgangspunkt seiner Analyse ist die Beobachtung, dass das „Opfer“ (,mark“) eines Trickbetrugs neben dem finanziellen Verlust immer auch einen Gesichtsverlust erleidet, der es in seinem Selbstbild bedroht, daher das Risiko eskalierender Reaktionen birgt und die Betrüger*innen zu präventiv deeskalierender Kommunikation veranlasst. Um das Opfer davon abzuhalten, eine Szene zu machen oder gar die Polizei einzuschalten, so Goffmans Beobachtung, wenden die Betrüger*innen verschiedene „Auskühlungsmaßnahmen" an, die dem Opfer eine selbstbildverträgliche Redefinition der Situation ermöglichen und es dadurch mit seinem Verlust versöhnen.

Der Trickbetrug stellt sicher eine sehr spezifische Konstellation der Selbstbildbedrohung dar. Wie verschiedene Studien gezeigt haben und wie Goffman selbst argumentiert, ist das Cooling-out-Konzept jedoch weitaus breiter anwendbar - schließlich ist das Leben in modernen Gesellschaften reich an Möglichkeiten, als Individuum an Erwartungen zu scheitern und infolgedessen einen „Rollentod“ (ebd., S. 462) zu erleiden. ${ }^{1}$ Dies gilt auch für das Beziehungsleben, das Goffman in seinem Aufsatz als Paradebeispiel für das Cooling-out in informellen Settings anführt. ,The problem of cooling persons out in informal social intercourse", so seine Bemerkung dazu, , is seen most clearly, perhaps, in courting situations and in what might be called decourting situations“ (ebd., S. 456). Drehen sich erstere Situationen darum, das Werbeverhalten einer Person, etwa in Form von Heiratsanträgen, negativ zu bescheiden, ${ }^{2}$ so betrifft letzteres die Auflösung einer Ehe, bei der einer Person die Rolle als würdi-

\footnotetext{
1 Für Anwendungen des Cooling-out-Konzepts auf so verschiedene Bereiche wie Bildung, Beruf, Wissenschaft, Leistungssport, Talentwettbewerbe, Familienbildung und Flirten siehe neben den anderen Beiträgen in diesem Schwerpunkt etwa Clark (1960), Nadai (2007), Thomas (2014), Snow et al. (1991) und Wei (2016).

2 Für eine Analyse dazu, wie Frauen unerwünschte Avancen von Männern im Nachtleben abwehren, vgl. Snow et al. (1991).
} 
ge Partner*in ${ }^{3}$ entzogen wird und sie dergestalt ,,ausgekühlt“ werden muss, dass sie die Scheidung akzeptiert, etwa indem sie das alternative Rollenangebot „Freund*in“ annimmt.

Knapp sieben Jahrzehnte nach der Veröffentlichung von Goffmans Analyse stellt sich die Frage, inwiefern das Cooling-out-Konzept für das Verständnis gegenwärtiger Trennungserfahrungen hilfreich ist. Schließlich entstand Goffmans Analyse in den 1950er-Jahren, dem ,golden age of marriage“, als nicht nur Eheschließungen, sondern auch die Stabilität von Ehen selbstverständlich waren. Scheidungen waren entsprechend ungewöhnlich und gingen für die Betroffenen nicht selten mit Stigmatisierungen einher, die eine Wiederaufrichtung des Selbst erforderlich machten. Heute ist die westliche Welt auf den ersten Blick eine andere: In Deutschland wird rund jede dritte Ehe geschieden (Statistisches Bundesamt 2018) und in den USA ist bei einer Scheidungsquote von 40 bis $50 \%$ gar von einer „Kultur der Scheidung“ die Rede (Ruspini 2013). Mit der Durchsetzung des Beziehungsmusters der seriellen Monogamie haben darüber hinaus die Trennungen unverheirateter Paare zugenommen (Schmidt et al. 2006, S. 26ff.). Zu dieser Normalisierung von Trennung und Scheidung kommt ihre Entproblematisierung und Entstigmatisierung: Rechtliche, religiöse und soziale Schranken sowie Sanktionen haben an Bedeutung verloren. So haben beispielsweise die Kirchen, die die Scheidung einst zur Sünde erklärten, nicht nur an Mitgliedern und gesellschaftlicher Relevanz eingebüßt, sondern durchlaufen auch gewisse Wandlungsprozesse. Während die evangelische Kirche Scheidungen zulässt, können katholisch geschlossene Ehen unter bestimmten Umständen in einem „Ehenichtigkeitsverfahren“ annulliert werden (Engler 2018). Auch die Einstellungen der Bevölkerung haben sich gewandelt. Moralisch erscheint es immer weniger verwerflich, eine Beziehung oder gar eine Ehe aufzulösen (vgl. z.B. Gerstel 1987; Zartler 2012, S. 68 f.). Im Gender and Generation Survey 2005 beispielsweise stimmten rund $82 \%$ der Befragten in Deutschland der Aussage „Es ist in Ordnung, wenn sich ein Paar in einer unglücklichen Ehe scheiden lässt, auch wenn sie Kinder haben“" sehr zu bzw. zu (Mühling und Schreyer 2012, S. 43). Könnte angesichts dessen heutzutage zutreffen, was Goffman (1952, S. $454 \mathrm{f}$.) in einem kurzen Einschub erwähnte, nämlich dass im historischen Zeitablauf die Tendenz zu beobachten ist, dass Verlust und Niederlagen zunehmend nicht moralisierend betrachtet werden? Träfe dies auch für Trennungen und Scheidungen zu, so würden diese immer weniger als moralisch zurechenbares ,Versagen“ der Einzelnen bewertet, sondern vermehrt lediglich als bedauernswerte Schicksalsschläge.

Auf Basis einer eigenen Studie zu Trennungen (Abschnitt 2) und von bereits veröffentlichten Studien zu Trennungs- und Scheidungserfahrungen aus dem deutschund englischsprachigen Raum zeige ich zunächst anhand einer Darstellung des gegenwärtigen Wissens zum Thema Trennungsfolgen, dass Trennungen für die Betroffenen nach wie vor eine Identitätsbedrohung darstellen (Abschnitt 3). Die Entwicklung einer neuen Selbst- und Wirklichkeitsdefinition stellt daher eine wichtige, wenn nicht die zentrale Aufgabe bei der Trennungsbewältigung dar. Entsprechend

\footnotetext{
3 Der Empfehlung der Zeitschrift entsprechend verwende ich im Folgenden das um ein Sonderzeichen erweiterte generische Femininum. So nicht anders markiert oder kontextuell naheliegend, sind also mit „Ex-Partner*innen“ immer auch „Ex-Partner“ mitgemeint.
} 
lässt sich das Cooling-out-Konzept, gerade auch mit Blick auf Goffmans spätere Analysen zur ,,beschädigten Identität“ (1975 [1963]), für die familiensoziologische Theoriebildung fruchtbar machen. Wie ich zeigen werde, liegt die Relevanz des Konzepts insbesondere darin, einen integrativen und genuin soziologischen Rahmen für verschiedene empirisch feststellbare materielle und vor allem symbolische Trennungs- bzw. Scheidungsfolgen, die damit einhergehenden interpretativen Krisen sowie deren Bewältigung zu bieten. Zugleich lädt die Beschäftigung mit dem Gegenstand des Beziehungsscheiterns zum Weiterdenken von Goffmans Coolingout-Konzept ein (Abschnitt 4).

Bei der Auswertung eigener qualitativer Interviews hat sich erstens gezeigt, dass das Cooling-out infolge von Beziehungsscheitern nicht als einmaliges, ritualhaftes Interaktionsereignis, sondern vielmehr als längerer Prozess zu begreifen ist, was in der Cooling-out-Literatur bislang nicht ausreichend berücksichtigt wurde. Zweitens lässt sich die von Goffman wohl in Analogie zum Trickbetrug nahegelegte Rollentrennung in die sich trennende, um Abkühlung bemühte Partner*in und die verlassene Partner*in als Opfer nicht aufrechterhalten: Trennungen bringen zwei Opfer hervor, die gemeinsam, alleine oder mit anderen nach auskühlenden Situations- und Selbstdeutungen suchen. Drittens werden abkühlende Deutungsrahmen nicht ad hoc und auf idiosynkratische Weise entwickelt. Vielmehr greifen die Akteure auf vorgefertigte Deutungsrahmen zurück, die milieuspezifisch geprägt sind. Die These der Milieuspezifizität des Cooling-out bei Trennungen wird exemplarisch anhand zweier Milieus veranschaulicht, die sich in ihrer sozialstrukturellen Stellung und ihren Deutungsmustern maximal voneinander unterscheiden (Abschnitt 5). Dies legt nicht nur nahe, Goffmans Cooling-out-Analyse mit seiner Rahmenanalyse (1980 [1974]) zu verbinden, sondern verweist auch auf die in Goffmans Cooling-out-Aufsatz (1952, S. 454) nur angedeutete und in der weiteren Cooling-out-Literatur nicht weiter untersuchte Relevanz sozialer Unterschiede bei der Wahl der Bewertungsregister und - hier im Fokus - der abkühlenden Deutungsrahmen (Abschnitt 6). Abschließend werden zentrale Erkenntnisse zusammengefasst und weitere Forschungsmöglichkeiten dargestellt (Abschnitt 7).

\section{Die Materialgrundlage}

Als eigene empirische Grundlage für die folgenden Ausführungen dienen Interviews aus dem 2018 abgeschlossenen Forschungsprojekt „Paare nach der Trennung“. ${ }^{4}$ Da Paare eine Realität sui generis ausbilden (Berger und Kellner 1965), stellte das Ex-Paar nicht nur in theoretischer, sondern auch in methodisch-empirischer Hin-

\footnotetext{
4 Das Projekt „Paare nach der Trennung“ (2016-2018) stellte die zweite Phase des DFG-geförderten Forschungsprojekts „Geschlechterarrangements in Paarbeziehungen im Milieuvergleich“ dar (zur ersten Phase von 2012 bis 2014 vgl. Koppetsch und Speck 2015). Während in der zweiten Projektphase die Frage nach den Ursachen des Beziehungsscheiterns im Vordergrund stand (vgl. dazu Eckert et al. 2021 und Koppetsch et al. 2019), fokussiere ich hier auf dessen Folgen und knüpfe dabei an Analysen zur Milieuspezifik von Trennungserzählungen an (Eckert et al. 2019). Cornelia Koppetsch und Eva-Maria Bub danke ich herzlich für die gemeinsame Arbeit im Projekt und besonders für die gemeinsamen Analysen, auf die ich hier teilweise zurückgreife.
} 
sicht die Analyseeinheit im Forschungsprojekt dar. Aus diesem Grund sollten beide Ex-Partner*innen für Interviews zur Verfügung stehen. Schlussendlich konnten wir über Interviewaufrufe u.a. in Graduierten-Netzwerken, der Bild-Zeitung, der taz, in Veranstaltungsmagazinen und - wegen einer Aufwandsentschädigung in Höhe von 50 Euro pro Person - in Nebenjobbörsen 23 Ex-Paare für unsere Studie gewinnen. Beide Ex-Partner*innen wurden getrennt voneinander in weitgehend teilnarrativ angelegten Einzelinterviews zur gesamten Beziehungsgeschichte vom Kennenlernen bis zur Trennung befragt. Erfragt wurden auch die eigene Herkunft sowie die Bildungs- und Berufsbiographie. Durchschnittlich dauerten die Interviews zwei Stunden und 15 Minuten. Die interviewten Ex-Paare hatten jeweils mindestens ein Jahr lang zusammengewohnt. Die Interviewpartner*innen decken eine Altersspanne von 25 bis 62 Jahren und ein breites Bildungs- und Berufsspektrum ab: Ungelernte Arbeiter*innen sind ebenso vertreten wie promovierte Akademiker*innen. 19 ExPaare lebten in einer gegengeschlechtlichen, drei in einer gleichgeschlechtlichen und eines in einer Beziehung, in der eine Person transitionierte. Sie waren zwischen drei und 35 Jahren zusammen, teils verheiratet und haben teils Kinder.

Die Auswertung erfolgte anhand einer Kombination unterschiedlicher rekonstruktiver Zugänge aus der Biographieforschung (vgl. v.a. Rosenthal 2014) sowie dem Integrativen Basisverfahren (Kruse 2015), die wir entlang der iterativ-zyklischen Forschungslogik der Grounded-Theory-Methodologie (Strauss und Corbin 1996) kombinierten. Insbesondere die Analyseheuristiken des Integrativen Basisverfahrens waren für die in diesem Beitrag vorgestellten Ergebnisse nützlich, v.a. die Positionierungsanalyse (vgl. dazu Lucius-Hoene und Deppermann 2004). Anhand dieser Heuristik konnten die Selbstpräsentation der Interviewpartner*innen und die Darstellung ihrer Ex-Partner*innen als moralisch ,gut“ oder „,böse“ sowie ihre narrative Konstruktion gegenwärtiger im Vergleich zu vergangenen Identitäten untersucht werden (vgl. ausführlicher dazu Eckert et al. 2019).

\section{Trennungsfolgen und Trennungsbewältigung: Beobachtungen der Familiensoziologie}

Um die familiensoziologische Relevanz des Cooling-out-Konzepts herauszuarbeiten, ist zunächst ein Einblick in den Forschungsstand zum Thema Trennung und Scheidung angezeigt. Von Trennung und Scheidung ist hier die Rede, da ältere und quantitative Studien in der Regel nur die Trennungen verheirateter Paare untersuchen und dabei die Scheidung als amtliche Auflösung einer Beziehung anstelle der vorherigen Trennung fokussieren (kritisch dazu Lenz 2006, S. 120f.).

\subsection{Trennungs- und Scheidungsfolgen}

Ungefähr seit der Jahrtausendwende hat sich der Fokus der Familienforschung von der Untersuchung der Scheidungsursachen auf die Analyse von Scheidungsfolgen verschoben (Hill und Kopp 2015, S. 221). Eine zentrale Erkenntnis der Scheidungsfolgenforschung ist dabei die folgende: ,Je höher der Institutionalisierungsgrad [der Beziehung], desto schwerwiegender sind in der Regel die ökonomischen Konse- 
quenzen und die psychischen Beeinträchtigungen nach einer Trennung und umso schwieriger gestaltet sich der Prozess der Ablösung vom Ex-Partner.“ (Peuckert 2019, S. 282)

In Bezug auf die materiellen Trennungs- und Scheidungsfolgen, die in der soziologischen Forschung bislang am intensivsten erforscht wurden (Kopp et al. 2010, S. 166), ist festzuhalten, dass eine Trennung im Regelfall für beide Ex-Partner*innen mit finanziellen Verlusten einhergeht: Bei gemeinsamer Haushaltsführung werden vormalige Einsparnisse obsolet und die Aufteilung der gemeinsamen Besitztümer macht nicht nur Neuanschaffungen erforderlich, sondern auch zumindest für eine Partner*in den Umzug in eine andere, meist kleinere Wohnung - oder den Verkauf der ehemals gemeinsamen Wohnung unter Wert (Weiss 1980). Hinzu kommen Einkommenseinbußen, die Frauen besonders treffen (Andreß 2003; Bröckel und Andreß 2015). Seit der Jahrtausendwende hat auch die Abhängigkeit Geschiedener von staatlichen Transfers zugenommen (ebd.). Mit den materiellen Verlusten steigen zugleich die psychosozialen Belastungen (Kopp et al. 2010, S. 179), was möglicherweise auf einen Statusverlust zurückzuführen ist: Infolge einer Scheidung beispielsweise zur Arbeitslosengeldempfängerin zu werden, kann als Stigmatisierung empfunden werden (Lenz 2006, S. 140).

Darüber hinaus identifizieren verschiedene Studien eine Reihe sozialer Belastungen. Dazu gehören anhaltende Konflikte mit der Ex-Partner*in, etwa um Sorgerechts- und Unterhaltsstreitigkeiten. Doch dabei bleibt es nicht: Im Vergleich zu Müttern verschlechtert sich bei Vätern der Kontakt zu den Kindern sehr viel stärker (Amato 2014, S. 10). Zudem halten ehemals gemeinsame Freund*innen und Bekannte je nach Loyalität den Kontakt zu nur einer Person und brechen ihn zur anderen Person ab. Außerdem nimmt der Kontakt von Getrennten zu Paaren ab (ebd.). All dies kann Vereinsamungsgefühle begünstigen und sich negativ auf das Wohlbefinden auswirken.

Hinsichtlich der psychischen und physischen Gesundheit zeigt sich, dass beide Partner*innen - also auch die Person, die die Trennung initiierte - über mehrere Jahre hinweg durchschnittlich stärker durch Versagensgefühle, Selbstzweifel, Schuld-, Reue- und Hassgefühle oder Depressivität belastet sind als Verheiratete (ebd.; Beelmann 1994, S. 56ff.; Werneck 2004). Diese psychischen Belastungen scheinen wiederum negative Effekte auf das Immunsystem zu haben, was Geschiedene anfälliger für Krankheiten verschiedener Art macht und in der Folge vermutlich zu ihrer erhöhten Krankheits- und Sterblichkeitsrate beiträgt (Filipp und Aymanns 2018, S. 90 ff.; Werneck 2004, S. 248).

Den (psycho-)sozialen und psychischen Trennungsfolgen kommt damit eine hohe Bedeutung für das generelle Wohlbefinden zu. Um sie präziser darstellen zu können, ist ein Blick über den Tellerrand der quantitativ geprägten Familiensoziologie nötig. Erforscht wurde diese Kategorie von Trennungsfolgen bisher nämlich vornehmlich in qualitativen (z.B. Hopper 2001; Riessman 1990; Sagstetter 1989; Schöningh et al. 1991; Singly 2011; Vaughan 1988; Weiss 1980) sowie psychologisch und therapeutisch orientierten Studien (Kopp et al. 2010, S. 167). 


\subsection{Sinn- und Selbstkrise}

Von besonderer Bedeutung ist in diesen Studien das Verständnis von Trennung als kritisches Lebensereignis (stressful life event), das - so die stresstheoretische Annahme - angesichts seiner negativen Folgen einen Stressor darstellt, aber - so die entwicklungspsychologische Perspektive - mittelfristig auch zum persönlichen Wachstum beitragen kann (vgl. Filipp und Aymanns 2018). Lebenslauftheoretisch betrachtet stellt die Trennung ein nicht-normatives Lebensereignis dar: Während normative Lebensereignisse wie der Schul- oder Renteneintritt vorhersehbar sind, viele betreffen und, wie etwa die Heirat, generell als erwünscht gelten, treffen nichtnormative Lebensereignisse die Individuen überraschend, gelten als unerwünscht und sind nicht mit definierten Bewältigungsmustern verbunden (Schöningh et al. 1991, S. 25). Ferner stellen kritische Lebensereignisse das Weltbild der Betroffenen infrage und erschüttern deren Selbstbild und Selbstwertbezug, etwa durch den „Entzug sozialer Positionen“, den „Ausschluss aus sozialen Bindungen oder den Verlust wichtiger Bezugspersonen, die der Selbstbestätigung dienlich waren“ (Filipp und Aymanns 2018, S. 62). Schließlich wirken sie sich potenziell negativ auf die sogenannte Zielrelevanz aus, insofern sie Handlungs- oder gar Lebensziele durchkreuzen, was sich wiederum negativ auf die Selbstdefinition auswirken kann (ebd., S. 56 ff.). Verschiedene qualitative Studien, darunter die eigene (Eckert et al. 2019), zeigen, was dies für Getrennte konkret bedeutet.

Berger und Kellner (1965) haben argumentiert, dass in Ehen - zeitgemäßer formuliert: in Paarbeziehungen - gemeinsam Wirklichkeit konstruiert wird. Trennungen lassen sich dementsprechend als umgekehrter Prozess verstehen: Der gemeinsame „nomos“ wird sukzessive aufgelöst und muss durch neue Weltsichten und Alltagsroutinen ersetzt werden (Singly 2011; Vaughan 1988; Weiss 1980; vgl. auch Herzer 2006). Dies kann zunächst zu einer Sinnkrise führen, die sich an der Deutung des Beziehungsscheiterns entzündet: Die Betroffenen fragen sich, wie es dazu kommen konnte. Dies gilt nicht nur für die verlassene Person, die mitunter von der Trennung überrascht wird, sondern trotz ihres Wissensvorsprungs (vgl. Vaughan 1988, S. $187 \mathrm{ff}$.) auch für die sich trennende Person. Obwohl sie unzufrieden war, kann sie die Gründe ihrer Unzufriedenheit nicht immer in Worte fassen. Für beide Ex-Partner*innen stellt sich daher die Aufgabe, eine Definition für die ungewisse Situation zu erarbeiten, was eine gewisse Zeit in Anspruch nimmt, wie z.B. Robert Weiss in seiner Studie beobachtete:

Gewöhnlich beschäftigen die Ereignisse, die zum Zusammenbruch geführt haben, die Gedanken der beiden getrennt lebenden Ehepartner noch Monate nach der Auflösung der Ehe. Wieder und wieder gehen sie in Gedanken durch, was schiefgegangen ist, rechtfertigen oder bedauern ihr Verhalten, wägen immer wieder ihre eigenen Worte und die des Ehepartners gegeneinander ab. [...] Ganz allmählich kommen sie dann mit den Ereignissen ihrer Ehe ins reine. (Weiss 1980, S. 38 f.)

Im Zuge dieser Infragestellung des Weltbilds wird auch das Selbst infrage gestellt (vgl. z.B. Hopper 2001). Mit Blick auf Berger und Kellners Analyse (1965) liegt dies daran, dass in Beziehungen auch Identitäten ausgebildet und stabilisiert werden. 
Eine Trennung bedeutet die Umkehrung auch dieses Prozesses: „Um auseinandergehen zu können, müssen die beiden Partner nicht nur ihren materiellen Besitz, sondern auch ihre Identitäten entflechten." (Vaughan 1988, S. 13) Wie de Singly (2011) zeigt, fällt diese Neudefinition der eigenen Person umso schwerer, je stärker die paarbezogenen, gemeinschaftlichen Anteile an der Identität ausgeprägt waren. Je mehr die eigene Identität hingegen durch beziehungsunabhängige Ich-Autonomie bestimmt wird, desto einfacher gestaltet sich die Trennung. Hinzu kommt in allen Fällen, dass die Ex-Partner*in als identitätsstabilisierende signifikante Andere wegbricht (Sagstetter 1989; Vaughan 1988). Die Entwicklung einer „nachehelichen“ Identität sowie deren interaktive Abstützung stellt daher eine zentrale Herausforderung dar (Weiss 1980).

Damit ist die durch Trennungen ausgelöste Identitätskrise aber noch nicht vollständig beschrieben. Vielmehr wird das Beziehungsscheitern auch als Scheitern des Selbst hinsichtlich gehegter oder antizipierter Erwartungen wahrgenommen:

Because personal relationships are almost universally viewed in success/failure terms, any party to a terminated or even a spoiled relationship is tarred by failure and - even more than the widowed or the orphaned - regarded as somehow odd, deficient, or deviant. (McCall 1982, S. 219; ähnlich Gerstel 1987, S. 173)

Dies gilt auch in Zeiten der eingangs angesprochenen öffentlichen Normalisierung, Entproblematisierung und Entstigmatisierung von Trennung und Scheidung, die im Privaten allerdings (noch?) nicht vollzogen sind. Nach wie vor gilt in großen Teilen der Bevölkerung eine feste Beziehung oder Ehe im Erwachsenenalter als erstrebens- und aufrechterhaltenswert. Vor diesem Hintergrund erscheinen Trennungen noch immer begründungspflichtig und werfen ein negatives Licht auf die Einzelnen (vgl. Doering 2010; Hopper 2001; Zartler 2012). Diese privatisierende Zurechnung des Beziehungsscheiterns wird dadurch verstärkt, dass von wenigen Ausnahmen abgesehen kaum institutionalisierte und leicht zugängliche Übergangsrituale und -skripte für die Passage von der ehelichen in die nacheheliche Beziehungsphase bzw. von der Beziehung in die Zeit danach existieren (Arosio 2016; Riessman 1990, S. $161 \mathrm{ff}$. .). ${ }^{5}$ Damit einhergehend stehen kaum positiv besetzte Rollen für Getrennte und Geschiedene zur Verfügung, sodass sich die Betroffenen latent in einer ,gesellschaftliche[n] Ausnahmeposition“"wähnen (Sagstetter 1989, S. 118). Die durch Trennungen ausgelöste Identitätsbedrohung wird für Eltern noch dadurch gesteigert, dass die intakte Kernfamilie nach wie vor als Idealfamilie gilt, wohingegen andere Familienformen unter dem Verdacht stehen, für das Wohlergehen der Kinder schädlich zu sein (Zartler 2012).

In der Folge sieht sich die Trennungsinitiator*in typischerweise mit der Schuldfrage konfrontiert, wie ich am Beispiel von Helen Schmahl, einer unserer Interview-

\footnotetext{
5 Von Übergangsritualen und -skripten unterscheiden sich die später vorgestellten milieuspezifischen Deutungsrahmen meines Erachtens dadurch, dass sie zwar Interpretationsangebote darstellen, aber weder eine konkrete Ausgestaltung des Übergangs (etwa durch eine Trennungsfeier analog zur Hochzeits- oder Trauerfeier) noch einen definierten Verarbeitungsfahrplan beinhalten. Dadurch wird die Trennungsbewältigung kaum kollektiviert, geschweige denn institutionalisiert.
} 
partner*innen, illustrieren möchte. ${ }^{6}$ Helen verließ ihren Mann Chris Schmahl, mit dem sie zwei Kinder hat, nach sechsjähriger Beziehung, nachdem sie sich in einen anderen Mann verliebt hatte:

HS: Ich hab immer sehr, sehr hohe Ansprüche gehabt an mich und wer ich sein wollte und das war zum Beispiel eine Person, die immer ehrlich und aufrichtig ist und nicht fremd geht, eine Person, die (.) eine liebevolle, gute Mutter ist (..) und ein treuer Partner ist äh (.) halt auch im Sinne von loyal, im Sinne von aufeinander bezogen und das (..) quasi, ich hab die Erwartungen von allen an mich und von mir selber an mich enttäuscht. ${ }^{7}$ (HS 2016)

Durch ihre Trennungsinitiative sieht Helen ihre angestrebten Identitätsfacetten als gute Mutter und gute Partnerin infrage gestellt und muss sich entsprechend wie andere Trennungsinitiator*innen mit dem realen oder imaginierten Vorwurf auseinandersetzen, egoistisch gehandelt und viel Leid verursacht $\mathrm{zu}$ haben (vgl. auch Doering 2010, S. 75):

HS: Ich hab auch ((schluchzend) wir haben immer uns ausgemalt, wie wir zusammen alt werden und so (...) ich hab diesen Traum aufgebaut mit ihm und hab ihn zerstört. Ich hab ihn weggenommen (.) ich hab ihm die Kinder weggenommen.) (HS 2016)

Für Verlassene rückt demgegenüber verstärkt die Scham in den Vordergrund, keine adäquate Partner*in gewesen zu sein (vgl. ebd., S. 74) - oder in den Worten von Chris Schmahl:

CS: Das Problem bei der ganzen Sache war, sie hat etwas beendet, was ich eigentlich noch für ziemlich gut hielt und äh (..) diese Traurigkeit führte dann dazu, dass ich mich gefragt habe, bin ich so ein furchtbarer Mensch, dass sie es nicht mehr mit mir aushält? Und bereit ist, all das, was wir haben, eben auch mit den Kindern, hinzuwerfen? (CS 2016)

Chris’ Kränkung, von Helen zurückgewiesen worden zu sein, wird noch dadurch verstärkt, dass er ihre neue Partnerwahl nicht nachvollziehen kann, da ihr neuer Partner ihn ,unterbietet“: Während Chris selbst zum Trennungszeitpunkt 28 Jahre alt, verheirateter Familienvater und in den Endzügen seines Studiums war, war Helens neuer Partner „22, hieß Justin und wohnte bei seiner Mutter“. Dass Helen Justin trotz seines jungen Alters, seines Namens mit geringem Sozialprestige

\footnotetext{
${ }^{6}$ Bei den Namen der Interviewpartner*innen handelt es sich um Pseudonyme; auch weitere personenbezogene Informationen sind verfremdet.

7 Die zitierten Transkriptpassagen wurden für die Publikation leicht geglättet. Das Prinzip der wortwörtlichen Abschrift blieb aber gewahrt. Weiterhin wurden starke Betonungen (Großbuchstaben) und die Länge der Pausen (in Klammern) beibehalten: Pausen kürzer als zwei Sekunden werden mit einem Punkt markiert, Pausen zwischen drei und fünf Sekunden mit zwei Punkten und Pausen länger als fünf Sekunden mit drei Punkten. Die zitierten Interviewpassagen enthalten ferner außersprachliche oder sprachbegleitende Handlungen wie Weinen, die in doppelten Klammern notiert sind: In der inneren Klammer wird die Handlung bezeichnet, während die äußere Klammer den Bereich markiert, über den sich die Handlung erstreckt.
} 
und seiner Unselbstständigkeit als Partner vorzieht, stellt für Chris eine zusätzliche Degradierung dar, die seine Selbstzweifel und Verunsicherung weiter steigern.

Insgesamt hat die Identitätskrise verschiedene Seiten, sowohl in Bezug auf die infrage gestellten Identitätsfacetten als adäquate Partner*in und gute Mutter bzw. guter Vater als auch hinsichtlich der Selbstwahrnehmung und der Identifizierung durch andere (vgl. Sagstetter 1989), denn ,[z]um Verlust an Selbstwertgefühl kommt der Verlust an Ansehen“ (Vaughan 1988, S. 172). Letzteres zeigt sich auch in den Herausforderungen bei der Gestaltung des Trennungs-,,Outings“, das sorgfältig vorbereitet wird, um Freund*innen oder Familienangehörige auf die eigene Seite zu ziehen (Gerstel 1987, S. 184).

Identitätsentwürfe zeichnen sich ferner dadurch aus, dass sie in die Zukunft projiziert werden. Eine trennungsinduzierte Identitätskrise lässt Pläne obsolet werden und stellt die Betroffenen vor die Aufgabe, Alternativen zu entwerfen, ihr Leben neu zu konzipieren (vgl. Singly 2011) und alltagspraktisch neu zu organisieren (vgl. Lenz 2006, S. 137; Weiss 1980, S. 370 ff.). Für Chris Schmahl beispielsweise stellt sich die Aufgabe, es Helen im „Entlieben“ gleich zu tun, mit ihr einen freundschaftlichen, respektvollen Umgang zu finden, sich selbst neu zu verlieben und die angestrebte neue Partnerschaft mit der Erziehung der Kinder vereinbaren zu können. Für eine Interviewpartnerin Mitte 50 stellen sich nach dem Ende einer 35-jährigen Beziehung die drei „Kernfragen“ „,wer bin ich, wo will ich hin und mit wem?“ (NU 2017), die sie der Reihe nach klären will. Eine andere Interviewpartnerin, Ende 30, sieht ihren zentralen Wunsch nach einer Familiengründung außer Reichweite, nachdem sie von ihrem Verlobten verlassen wurde:

CE: Es ist klar sehr schmerzhaft, jetzt fang ich wieder an zu heulen ((weinen) dass ich nicht weiß, wie's mit Partnerschaft ist, und dass ich halt nicht mehr so jung bin. Das war halt irgendwie schon mein erster Gedanke, dass ich dachte, das nach all der Zeit, das war schon das Gefühl, dass ich dachte, jetzt hab ich so lang gewartet, jetzt könnt's losgehen, jetzt) war's, also da war ich knapp acht und oder siebenunddreißig noch und so ne Verarbeitung braucht Zeit, ich bin nicht jemand, der sich schnell wo anders reinstürzt, ich bin, das macht auch kein Sinn, kann man auch nicht forcieren und das ist halt das Gefühl, ich weiß nich, ((weinend) ob meine Träume noch erfüllt werden). (CE 2017)

\subsection{Trennungsbewältigung}

Die beschriebenen Sinn-, Selbst- und Planungskrisen lassen sich auch als interpretative Probleme fassen, die von den getrennten Personen auf je individuelle Weise zu lösen sind (Hopper 2001). Wie zahlreiche Forschungen zur Bewältigung von Trennungen und zur Anpassung an die neue Lebenssituation zeigen, ist eine gelingende Problemlösung nicht nur von verfügbaren Ressourcen - darunter sozialer Unterstützung, auf die ich noch näher eingehen werde -, sondern auch von subjektiven Redefinitionen abhängig (Amato 2000, 2014; Arránz Becker 2015; Gray und Silver 1990; Kopp et al. 2010; Wang und Amato 2000).

Hinsichtlich des Beziehungsscheiterns geht es für die Betroffenen darum, zu verstehen, wie es zur Trennung kam, und das komplexe Geschehen in eine für sie 
sinnhafte und in der Regel zeitlich linearisierte Gestalt zu bringen, die es ihnen erlaubt, Kontrolle zurückzugewinnen und die Trennung als abgeschlossenen Teil der eigenen Vergangenheit zu betrachten (Weiss 1980). Wer sich und anderen die Trennung erklären kann, fühlt sich weniger belastet, wobei zu gelten scheint: ,Je mehr subjektive Ursachen insgesamt genannt werden, desto geringer fällt die Belastung nach der Trennung aus“ (Kopp et al. 2010, S. 183). Auch die Art und Weise der Ursachenzuschreibung ist bewältigungsrelevant, wie Studien zeigen, die mit Varianten der Attributionstheorie ${ }^{8}$ (Grych und Fincham 1992) oder mit dem Account-Konzept ${ }^{9}$ (Hopper 2001) arbeiten. Entgegen der attributionstheoretischen Erwartung, dass sich eine Externalisierung der Trennungsverantwortung positiv auswirkt, zeigt sich, dass eine reine Opferrolle die Anpassungsfähigkeit beeinträchtigt (Amato und Previti 2003; Gray und Silver 1990) - genauso wie das Fehlen einer Erklärung: „Having a sense of being a victim and having no explanation for the event may contribute to a focus on the past that may interfere with the ability to move forward with one's life" (Gray und Silver 1990, S. 1189). Am besten gelingt die Anpassung denjenigen, die gewisse Handlungsspielräume hinsichtlich einer Kontrollmöglichkeit über das Geschehen bei sich selbst sehen und die Trennungsursache in der Beziehung, insbesondere in der Nicht-Passung der beiden Partner*innen, verorten. Dadurch erscheint ihnen die Trennung retrospektiv unvermeidbar (Amato und Previti 2003, S. 620; Kopp et al. 2010, S. 183; vgl. auch Grych und Fincham 1992).

Für eine solche Ursachenzuschreibung stellt das tatsächliche Beziehungs- und Trennungsgeschehen zwar den Ausgangsstoff dar. Allerdings bestehen einige interpretative Freiheiten, bestimmte Situationen zu dramatisieren und andere auszusparen (Weiss 1980, S. 39) sowie bestimmte Rollen zu konstruieren (Hopper 2001). Im Ergebnis werden Trennungsgeschichten in einer „biased and ego-enhancing fashion“ (Gray und Silver 1990, S. 1180) erzählt. Accounts stellen daher stets auch ,facesaving devices“ (Riessman 1990, S. 14) dar, die Schuld abwenden, ihre Erzähler*in im Diesseits der moralischen Ordnung situieren und der Ex-Partner*in die Rolle der Bösen zuschreiben. Dies ist insofern wichtig, als die Trennungsbewältigung auch eine erfolgreiche Identitätsrekonstruktion erfordert: ,Successful adjustment also requires people to develop new identities that no longer are bound up with the former marriage“" (Amato 2014, S. 11). Relevant hierbei scheint, dass neben der Vergewisserung, moralisch richtig gehandelt und sich nichts vorzuwerfen zu haben (Hopper 2001), ein konsistentes Selbst in der diskontinuierlichen Zeit des Beziehungsscheiterns konstruiert wird (Filipp und Aymanns 2018, S. 205 ff.).

Neben der erfolgreichen subjektiven Redefinition des Beziehungsscheiterns und des Selbst trägt das Vorhandensein von Ressourcen zu einer erfolgreichen Bewältigung bei, wobei an erster Stelle soziale Unterstützung zu nennen ist (vgl. Wang und Amato 2000, S. 666f.; sowie Filipp und Aymanns 2018, S. 233 ff. in Bezug

\footnotetext{
8 Attributionstheorien sind in der Psychologie beheimatet und dienen dazu, individuelle Kausalerklärungen von menschlichem Verhalten zu untersuchen - z.B. ob dieses internal oder external zugerechnet wird.

9 Das Account-Konzept wurde von Scott und Lyman bekannt gemacht und kann folgendermaßen umrissen werden: ,Scott and Lyman define an account as ,a linguistic device employed whenever an action is subjected to valuative inquiry'. Accounts are used to explain behavior that is ,bad, wrong, inept, unwelcome, or in some other of the numerous possible ways, untoward“" (Wei 2016, S. 5 f.).
} 
auf kritische Lebensereignisse generell). Dass Frauen häufig über bessere Unterstützungsnetzwerke verfügen als ihre Ex-Partner, kann ein Grund dafür sein, dass ihnen die Anpassung an die neue Lebenssituation in der Regel besser gelingt (Hill und Kopp 2015, S. 223). Auch eine neue Beziehung erleichtert die Trennungsbewältigung (Wang und Amato 2000, S. 666f.). Worauf genau die positiven Effekte sozialer Beziehungen beruhen, ist allerdings noch zu klären. In der Forschung zu kritischen Lebensereignissen dominierten bislang die Analyse konkreter Unterstützungsleistungen und die Frage ihrer taxonomischen Ordnung im Sinne emotionaler, informationeller bzw. kognitiver und instrumenteller Unterstützungsformen (vgl. Filipp und Aymanns 2018, S. 261 f.).

Der dargelegte Forschungsstand zum Thema Trennungsfolgen und -bewältigung zeigt, dass nach Trennungen neben ökonomischen Problemen vor allem interpretative Probleme bewältigt werden müssen. Während das gängige familiensoziologische Theorieinstrumentarium insbesondere mit Ansätzen im Anschluss an Gary Becker ökonomische Probleme gut fassen kann (Kopp et al. 2010, S. 186), werden interpretative Probleme wenn überhaupt meist nur anhand psychologischer Konzepte gefasst. $\mathrm{Zu}$ letzteren gehören unter anderem stresstheoretische Ansätze (Amato 2000; Andreß 2003, S. 280 ff.; Hill und Kopp 2015, S. 222) und das Konzept der kritischen Lebensereignisse (Sagstetter 1989; Schöningh et al. 1991). Im Unterschied dazu genuin soziologischer Art, eignet sich Goffmans Cooling-out-Konzept als integratives Instrumentarium für unterschiedlichste Verlust- und Krisenerfahrungen und damit einhergehende Bewältigungsaufgaben (vgl. auch Usera 2018). Es schärft den Blick für die vielgestaltigen interpretativen Krisen - von Sinn- über Selbst- bis hin zu Planungskrisen -, mit denen sich Ex-Partner*innen nach einer Trennung konfrontiert sehen. Wie in diesem Beitrag gezeigt wird, gilt dies zumal dann, wenn man das Konzept um weitere Ansätze aus Goffmans Werk erweitert.

\section{Cooling-out in Trennungskrisen: Konzeptionelle Grundlagen}

Wie in Abschnitt 3.2 gezeigt, stellt sich für die Getrennten die Aufgabe, das Geschehene zu ordnen und die gescheiterte Beziehung neu zu definieren. Die zweite zentrale Bewältigungsaufgabe stellt die ,Reparatur“ der, mit einem späteren Begriff Goffmans (1975 [1963]) gesprochen, ,,beschädigten Identität“ dar. Es ist anzunehmen, dass der „Reparaturbedarf“ mit der Anzahl und Bedeutung der Rollen, die die Akteure innehatten, steigt: Je institutionalisierter die Beziehung, desto mehr „Rollentode" drohen (Goffman 1952, S. 462), desto mehr wird das Beziehungsscheitern begründungspflichtig (vgl. Schneider 1990) und desto stärker fällt die Identitätskrise aus. So fragt sich Helen Schmahl nicht nur, ob sie eine gute Partnerin, sondern auch, ob sie eine gute Mutter sein kann. Wie gut diese interpretativen Herausforderungen bewältigt werden, hat nach aktuellem Forschungsstand weitreichende Folgen für die psychische und physische Gesundheit der Betroffenen.

Im Einklang mit der empirischen Literatur steht Goffmans Annahme, dass die Lösung dieser interpretativen Krisen nur durch eine Situations- und Selbstredefinition erfolgen kann, deren Herstellung nicht nur psychische, sondern auch soziale Ressourcen erfordert. Die betroffene Person benötigt ,a new set of apologies for 
himself, a new framework in which to see himself and judge himself" (Goffman 1952, S. 456). Um dieses „framework“ zu konstruieren, ist sie auf soziale Unterstützung durch jene angewiesen, die bei Goffman ,cooler“ heißen. Der in der Literatur wiederholt festgestellte positive Einfluss sozialer Unterstützung auf die Bewältigung der Trennung ist folglich darauf zurückzuführen, dass Freund*innen und andere „cooler“ der betroffenen Person als geduldige Zuhörer*innen, verständnisvolle Rezipient*innen und engagierte Co-Produzent*innen ihrer Neudeutungsversuche zur Seite stehen (vgl. auch die entsprechenden Hinweise bei Filipp und Aymanns 2018, S. 17 f., 278; sowie Sagstetter 1989, S. 141).

Über diese allgemeine, familiensoziologisch fruchtbare Konzeptualisierung hinaus bietet Goffmans Beschreibung der Interaktionssituation von ,cooler“ und „mark“ jedoch noch weitere vielversprechende Ansatzpunkte. Neben diversen Varianten der „Auskühlung“ erwähnt Goffman auch Fälle, in denen die Betrogenen die „Auskühlungsversuche“ zurückweisen, und verweist auf ihre Handlungsoptionen, um den „Trostprozess“ zu vermeiden. In Bezug auf die genutzten „Auskühlungsstrategien“ diskutiert er die Externalisierung der ,cooler“-Rolle an andere Personen wie Freund*innen des Opfers, das Anbieten eines alternativen Status der Freund*in anstelle der Partner*in oder das Gewähren einer weiteren Chance, um sich für die infrage stehende Rolle zu qualifizieren.

Daniel Usera (2018) kommt das Verdienst zu, die vorliegende Forschungsliteratur zu Trennungen eng entlang dieser Goffman'schen Anregungen durchforstet zu haben. Dabei erweitert er die von Goffman identifizierten Strategien punktuell und kommt in seinem Ausblick auf einige Begrenzungen dieser analytischen Perspektive zu sprechen (ebd., S. 17). So merkt er an, dass Goffman nicht eindeutig definiere, wann der Cooling-out-Prozess startet und wann er endet. Während Usera als Kommunikationswissenschaftler auf die Sprechakte in der Konversation des Paares fokussiert, sieht er gleichwohl in der Konstellation, die sich nach diesem Gespräch einstellt, ein noch umfangreicheres Anwendungsfeld für Goffmans Konzept. Weiterhin diskutiert er, dass das von Goffman untersuchte Setting - eine Person ist unzufrieden, will die Beziehung beenden und muss die andere Person auskühlen zu unterkomplex und linear gedacht sein könnte, etwa da sich die Rollen zwischen den beiden Partner*innen angesichts der Dynamik des Beziehungslebens ändern könnten. Diese Gedanken möchte ich aufgreifen und auf Basis der eigenen empirischen Studie Überlegungen anstellen, inwiefern die Analyse des Umgangs mit dem Scheitern von Paarbeziehungen zur Weiterentwicklung des Cooling-out-Konzepts beitragen kann.

Erstens ist es, wie bereits von Usera angedacht, angemessen, das Cooling-out nicht als einmaliges Interaktionsereignis, sondern als längeren Prozess zu betrachten, der in zeitlicher Hinsicht weit - teils Jahre - über den Vollzug der Trennung oder den Verwaltungsakt der Scheidung hinausreicht. ${ }^{10}$ Dies liegt u.a. daran, dass die Ursachen des Beziehungsscheiterns für die Akteure selbst teils unklar sind und anhand repetitiven und ruminativen (d.h. wiederkäuenden) Denkens und Kommunizierens Schritt für Schritt (re-)konstruiert werden (vgl. z.B. Weiss 1980). In diesem

10 Vgl. zur Prozesshaftigkeit des Cooling-out auch Thomas (2014, S. 296), der den Umgang von Müttern mit der Down-Syndrom-Diagnose ihres Kindes untersucht hat. 
Prozess spielen auch andere „,cooler“ als die Ex-Partner*in eine wichtige Rolle und werden zu Co-Produzent*innen der neuen Situations- und Selbstdefinition.

Zweitens kann es zwar zu Rollenwechseln in Bezug auf die Beziehungsunzufriedenheit und intendierte Trennungsinitiation kommen. Unabhängig davon, wer die Trennung am Ende aussprach, handelt es sich bei gescheiterten Beziehungen jedoch um ein Spiel mit zwei Verlierer*innen, die beide nach der ersten Trauerphase hoffen, im Laufe der Zeit zu Gewinner*innen zu werden, indem sie eine neue, „bessere“ Beziehung eingehen - eine von Goffman (1952, S. 459) erwähnte Variante der ,phantasies of the ,I'll show them“ kind“. Insofern bringt die Auflösung einer Paarbeziehung zwei „marks“ und mindestens zwei „,coolers“ hervor: Sowohl die sich trennende als auch die verlassene Person bedürfen nicht nur der Tröstung, sondern auch der Herstellung eines neuen „framework“ für die Redefinition ihrer Identität. Dass die sich trennende Person einen „Vorsprung“ (Vaughan 1988, S. 187 ff.) im Cooling-out-Prozess hat, da sie sich auf das Leben nach der Beziehung bereits vorbereiten konnte, ist in zeitlicher, aber nicht in prinzipieller Hinsicht relevant.

Drittens werden diese Redefinitionen des Selbst und der Situation nicht spontan und idiosynkratisch erschaffen, sondern folgen sozial differenzierten, genauer milieuspezifischen Deutungsmustern, was in der Cooling-out-Literatur bislang unbeachtet blieb. Zwar hat Goffman erwähnt, dass es neben einem zeitlichen Wandel eine soziokulturelle Variabilität in den Bewertungsregistern geben kann (1952, S. 454f.), diesen Aspekt aber - ähnlich wie die an ihn anschließende Cooling-out-Forschung nicht vertieft. Einzig Nadai (2007, S. 455) macht darauf aufmerksam, dass in ihrer Studie zur betrieblichen Aussortierung von ,Leistungsschwachen“ die Auswahl abkühlender Techniken auch ,,von strukturellen und kulturellen Merkmalen des Unternehmens" abhängt. Diese Leerstelle verwundert, da bekannt ist, dass es unterschiedliche Scheiternskulturen gibt (Backert 2004), und da in so verschiedenen Feldern wie beispielsweise der Ungleichheits-, Beziehungs- oder Geschlechterforschung seit Jahrzehnten klassen-, milieu- und geschlechterspezifische Wahrnehmungs-, Bewertungs- und Handlungsmuster herausgearbeitet werden (Bourdieu 1982; Koppetsch und Burkart 1999). Entsprechend finden sich auch in der Trennungsforschung einige Analysen zu Unterschieden in den Identitäts(re)konstruktionen und Trennungsdeutungen. Riessman (1990, S. $21 \mathrm{ff}$.) sowie Walzer und Oles (2003) beispielsweise haben diesbezüglich gezeigt, dass es geschlechtsspezifische Unterschiede dergestalt gibt, dass Männer und Frauen auf unterschiedliche kulturelle Vorstellungen einer guten Beziehung zurückgreifen. In unserem Sample von 23 Ex-Paaren erwiesen sich bei der Wahl der auskühlenden Situations- und Selbstdeutungsangebote allerdings weder das Geschlecht noch das Alter bzw. die Lebensphase oder der Institutionalisierungsgrad der Beziehung als zentral, sondern vielmehr die Milieuzugehörigkeit der Interviewpartner*innen, die hier in Anlehnung an Bourdieus Klassentheorie (1982) und die darauf aufbauenden Überlegungen von Koppetsch und Burkart (1999) rekonstruiert wurde (Eckert et al. 2019). 


\section{Soziale Unterschiede im Umgang mit dem Beziehungsscheitern: Cooling-out im individualisierten und im traditionalen Milieu}

Bourdieu sowie Koppetsch und Burkart folgend ist die Milieuzugehörigkeit durch die jeweilige Standortgebundenheit geprägt. Ausschlaggebend für die Verortung im „sozialen Raum“ sind soziale Lagen und damit verknüpfte Erfahrungen, die sich in habitualisierten milieuspezifischen Deutungs- und Handlungsmustern manifestieren. Spezifische Ausprägungen des ökonomischen, sozialen und kulturellen Kapitals, über das Akteure verfügen, korrespondieren demzufolge mit bestimmten Lebensstilen und kulturellen Leitvorstellungen - auch zu Geschlecht -, ohne diese zu determinieren (ausführlicher zur Milieukonzeption: Koppetsch und Burkart 1999, S. 285 ff.; vgl. auch Koppetsch und Speck 2015).

Dass die Milieuzugehörigkeit für die jeweilige Ausgestaltung der Cooling-outProzesse von hoher Relevanz ist, lässt sich anhand des Interviewmaterials gut zeigen. Die Milieubezeichnungen von Koppetsch und Burkart (1999) aufgreifend, spreche ich im Folgenden von zwei Milieus, denen sich diverse Interviewbeispiele zuordnen lassen: dem individualisierten und dem traditionalen Milieu. Die Konstruktion dieser Milieus folgt der Beobachtung, dass im Interviewsample die Unterschiede in der sozialstrukturellen Stellung mit Unterschieden in den jeweils typischen Deutungsmustern korrelieren, wobei sich diese Deutungsmuster maximal voneinander unterscheiden. Für beide Milieus liegt eine ausreichende Anzahl an Fällen vor, um von einer theoretischen Sättigung der milieuspezifischen Deutungsmuster ausgehen zu können. ${ }^{11}$ Nach der eher deskriptiv orientierten Darstellung der beiden Milieus stelle ich die daraus folgenden Schlussfolgerungen für das Cooling-out-Konzept dar.

\subsection{Das individualisierte Milieu: Helen und Chris Schmahl}

Das individualisierte Milieu (Koppetsch und Burkart 1999, S. 145 ff.; Koppetsch und Speck 2015, S. 55 ff.) zeichnet sich in sozialstruktureller Hinsicht in der Regel dadurch aus, dass seine Vertreter*innen akademisch gebildet sind und gemäß dem milieuspezifisch hohen Wert der Selbstverwirklichung typischerweise in Berufen arbeiten, die diesem Ideal entsprechen, ob in der Wissenschaft, dem Grafikdesign oder der Erlebnispädagogik. Selbstverwirklichung spielt aber auch in der Freizeitgestaltung eine große Rolle, vom Musikmachen bis hin zu einem insgesamt ,alternativen“ Lebensstil. Die Milieuzugehörigen leben vor allem in Großstädten, aber auch in alternativen Wohnformen im ländlichen Raum. Teil des Beziehungsleitbilds dieses Milieus sind die Egalität der beiden Partner*innen und in heterosexuellen Beziehungen die Gleichheit der Geschlechter.

\footnotetext{
11 Im Sample sind zwar auch einige Angehörige weiterer Milieus vertreten, aufgrund der geringen Fallzahl sind jedoch keine gesättigten Milieubeschreibungen möglich.

12 Die paarbezogene Darstellung bedeutet selbstverständlich nicht, dass es nur milieu-homogame Paare und Ex-Paare gäbe. Zwei Ex-Partner*innen zu Wort kommen zu lassen hat jedoch den Vorteil, unterschiedliche Redefinitionen einer geteilten Beziehungs- und Trennungsgeschichte darstellen zu können (vgl. auch Gray und Silver 1990).
} 
Als Beispielpaar für dieses Milieu eignet sich das bereits kurz vorgestellte Ex-Paar Helen und Chris Schmahl. ${ }^{12}$ Helen und Chris lernten sich mit Anfang 20 kennen. Schon früh erfolgte die Verlobung, die Geburt eines gemeinsamen Kindes und im dritten Beziehungsjahr die Heirat, die auch dazu diente, dass Chris Helens Kind aus einer früheren Beziehung adoptieren konnte. Paargeschichtlich ebenfalls früh fand die durch Helen initiierte Beziehungsöffnung statt, die Chris zunächst kränkte, von der er dann aber wie Helen Gebrauch machte. Zugleich mehrten sich die Konflikte um die Haushaltsführung, die Kindererziehung und die Finanzen. Nachdem sich Helen in einen anderen Mann, Justin, verliebt hatte, trennte sie sich von Chris. Die Interviews mit Helen und Chris fanden rund ein halbes Jahr nach der Trennung statt. Wie stellen Helen und Chris nun sich und ihre Geschichte im Interview dar?

Bei Helen ist auffällig, dass sie sich bereits im Vorfeld des Interviews als ,die Böse“ (HS 2016) vorstellt, die alles kaputt gemacht habe und sich ihrer Trennungsschuld bewusst ist. Damit nimmt sie die typische Zuschreibung an Trennungsinitiator*innen vorweg und widerlegt sie im Interview, indem sie ihre Handlungsmacht relativiert. Dies geschieht vor allem unter Rekurs auf Emotionen, die scheinbar schicksalhaft auftreten und im individualisierten Milieu als ,,authentische Signifikanten“ (Frevert 2009, S. 186) gelten, d.h. als ernstzunehmende Hinweise auf eigene Bedürfnisse und Richtschnur für weiteres Handeln, das im Einklang mit diesen Bedürfnissen steht. ${ }^{13}$ So formuliert Helen in Bezug auf die gesamte Beziehung: „Es waren immer Zweifel in irgendeiner Form da.“ (HS 2016) Für den weiteren Beziehungsverlauf konstatiert sie, dass ihre Gefühle zu Chris „,weniger“ geworden und ,verloren“ gegangen seien und sie darüber hinaus ,überhaupt nur so'n Schatten [war] von dem, was ich gerne wäre“. Statt ein authentisches, mit den eigenen Bedürfnissen und Zielen in Einklang stehendes Leben der Einzelnen zu fördern, wird die Beziehung dadurch zur Authentizitätsbedrohung. Ihr Beziehungsunglück wird laut Helen durch die Liaison mit Justin aufgefangen; Chris, der sich dagegen wendet, hält sie in ihrer Reinszenierung entgegen: „Ich kann das jetzt nicht aufgeben, weil ich jetzt wieder anfange, glücklich zu sein“. Am Ende entscheiden ihrer Darstellung nach die Gefühle zu Justin über ihre Trennung. Die neue Beziehung überbietet die alte, hier in emotionaler Hinsicht, was wiederum auf die handlungslegitimierende Funktion von Gefühlen im individualisierten Milieu hinweist.

Helens Selbstentschuldung dient auch ihre Erzählung über ihre Bemühungen, das Richtige zu tun und die Beziehung zu retten, da sie hierüber signalisiert, dass sie die Beziehung nicht leichtfertig aufgab. So führt sie an, dass sie kinderfreie Paarabende initiiert habe, aber mit Bedauern feststellen musste, dass sie und Chris nur darüber redeten, „was die Kinder Süßes gemacht haben“ (HS 2016), d.h. keine tiefergehende Kommunikation darüber erfolgte, was die beiden eigentlich beschäftigte. Auch zum Trennungszeitpunkt sinniert sie darüber, was die Beziehung hätte retten können, wobei sie sich selbst in die Verantwortung nimmt: „Ich spiel die ganze Zeit,was

\footnotetext{
13 Der Begriff der Authentizität wird weder von Helen noch von anderen individualisierten Interviewpartner*innen verwendet, sondern stellt ein von uns Forschenden rekonstruiertes Konzept dar, das ihren Interviewausführungen zugrundeliegt - auch solchen Äußerungen, die sich auf eine gelingende Beziehung beziehen. So ist in den Interviews u.a. davon die Rede, dass in einer solchen Beziehung ,,jeder sich selbst treu bleibt“", man ,sich selber nicht verliert“ und ,einfach Ich sein kann“.
} 
wäre wenn?`, was wäre gewesen, wenn ich früher in Therapie gegangen wäre? Vielleicht hätt ich [...] diese ganze Dynamik irgendwie stoppen können.“ Inmitten dieser Schuldaneignung, die das Interview dominiert, vermag sie es aber auch, Chris Schuld zuzuweisen, wenn sie sagt: „Ich hab das Gefühl, er war nicht immer ein guter Partner, aber trotzdem hätte ich besser sein müssen (..) und dass ich ihn mit seinen Fehlern hätte nehmen müssen.“

Ähnlich wie Helen vermeidet auch Chris eine offensive Schuldzuweisung, indem er Kritik an Helen nicht in den Vordergrund rückt. Nur nebenbei kennzeichnet er Helens Vorstellung an die Beziehung als „Disneyvorstellung“ (HS 2016) und wertet sie damit als unrealistisch ab. Im Vordergrund seiner Trennungserklärung steht hingegen ein allgemeines Passungsproblem mit Blick auf die unterschiedlichen Beziehungsvorstellungen der beiden:

CS: Irgendwann kamen wir dann zu dem Schluss, kam ich dann zu dem Schluss, ich bin tatsächlich kein furchtbarer Mensch, aber ich bin halt einfach nicht der Mensch, der für SIE geeignet ist. (CS 2016)

Angesichts dieser Inkompatibilität erscheint nicht nur die Schuldfrage obsolet. Vielmehr leuchtet, wenn man eine gelingende Beziehung als Authentizitätsgarantie ansieht, unmittelbar ein, warum unter diesen Bedingungen die Liebe zum Scheitern verurteilt ist: Sie ermöglicht es einer Person oder gar beiden Personen nicht, authentisch zu leben. Zugleich räumt Chris eigene Fehler ein, markiert sie aber als Ausnahme, beispielsweise in der Darstellung dessen, wie er auf Helens Ankündigung, die Beziehung zu beenden, reagierte:

CS: Ich war schockiert in dem Moment. Es hat mich natürlich auch verletzt. (.) Und (...) ich hab (..) ich bin nicht den Ansprüchen gerecht geworden, die ich sonst an mich habe. Also von meiner reflektierten (.) Reflektionsfähigkeit et cetera, sondern ich war wirklich sehr verletzt und hab vielleicht auch Dinge gesagt, die (..) ich heute auf gar keinen Fall wiederholen würde. (.) Ich könnt jetzt nicht mal mehr sagen, was es genau war, aber es war, es war (.) ich hab den neuen Partner, ohne ihn wirklich zu kennen, in dem Moment aus Eifersucht oder so schlecht zu machen. Zugegeben, er ist 22, hieß Justin und wohnte bei seiner Mutter, also es gab schon gewisse Ansatzpunkte sich darüber I: aufzuregen

CS: ja, und (.) mich da'n bisschen lustig drüber zu machen, war jetzt auch nicht so schwer. (CS 2016)

In dieser wie in anderen Äußerungen reklamiert Chris die Position des selbstreflektierten Menschen für sich, der über sein Agieren nachdenkt und an sich arbeitet. Fehler wie seine starke, klassistische Züge tragende Abwertung von Helens neuem Partner Justin werden in ihrer Bedeutung dadurch relativiert, dass sie reflexiv aufgearbeitet werden. Damit entwirft Chris eine anders konturierte Identität als Helen: Seine Richtschnur des Handelns sind nicht Emotionen wie Eifersucht, sondern die Reflexion, womit er einen anderen zentralen Wert des individualisierten Milieus für seine Identitätskonstruktion in Anspruch nimmt. Sowohl Helen als auch Chris eint aber, dass sie beide zugleich eine Konstanz und eine Weiterentwicklung ihres Selbst 
markieren: Während Helen daran arbeitet, emotional noch authentischer zu leben, bemüht sich Chris, noch reflektierter zu handeln.

Zusammenfassend und zugleich über dieses Fallbeispiel hinaus generalisierend lässt sich für das individualisierte Milieu festhalten, dass Trennungen als Resultat eines Authentizitätsmangels in der Beziehung gedeutet werden: Die Beziehung stellt für eine Partner*in oder - im Falle der diagnostizierten Nicht-Passung der Partner*innen - für beide ein Authentizitätshindernis dar. Ergänzend werden Kommunikationsprobleme genannt, vor allem ein Mangel an authentischer Kommunikation, in der sich die Partner*innen einander hinsichtlich ihrer Gefühle, Bedürfnisse und Sorge offenbaren. Eine solche Kommunikation erscheint den Beteiligten als bedeutsam, weil nur durch sie Konflikte als Stellvertreterkonflikte erkannt werden können. Um den wahren Charakter eines Konflikts gemeinsam zu entlarven, reicht es nicht aus, sich auf das Augenscheinliche, also auf konkrete Ereignisse und Handlungen, zu konzentrieren. Diese stellen aus Sicht der Beteiligten nur die „Oberfläche“ dar. Als viel wichtiger für das Verständnis von individuellen Problemen und Beziehungsproblemen gilt der „Rieseneisberg, der unten dranhängt“ (CE 2017), und ,was so im Unterbewusstsein so lauert“ (KA 2017), wie andere Interviewpartner*innen meinen. ${ }^{14}$

Als „Primärzugang“ zum Selbst und seinen auch unbewussten Wünschen gelten folglich in diesem Milieu die eigenen Emotionen. Kommunikation und Reflexion sind Mittel der Durchdringung des „Eisbergs“. Eine eigene positive Post-TrennungsIdentität wird dadurch hergestellt, dass die Trennung als Reflexionsanlass genommen wird, um sich selbst und die Geschehnisse besser zu verstehen. Dies ermöglicht es, die eigenen Prägungen und Schwächen zu erkennen und an einem besseren Selbst $\mathrm{zu}$ arbeiten - was es auch erlaubt, authentischer als zuvor zu leben und zu lieben. Auch das Interview wird von den individualisierten Interviewten als eine Etappe dieses Reflexionsprozesses verstanden und teilweise als „therapeutisches Gespräch“ betitelt, d.h. als Ort der Reflexion für sich und für deren Verbalisierung vor einem aufmerksam zuhörenden Publikum. Darüber hinaus dient es als säkularisiertes Beichtgespräch, in dem nicht die Ex-Partner*in auf offensive Weise beschuldigt, sondern zumindest rhetorisch Schuld angeeignet und Besserung gelobt wird, um das moralische Selbst zu rehabilitieren.

\subsection{Das traditionale Milieu: Jeanette und Sascha Schreiner}

Ganz anders gestalten sich die trennungsbezogenen Deutungsmuster im traditionalen Milieu (vgl. Koppetsch und Burkart 1999, S. 29 ff.; Koppetsch und Speck 2015, S. 93 ff.). Dessen Vertreter*innen üben großteils die klassischen Arbeiter*innenbzw. Ausbildungsberufe aus. Im Zuge der allgemeinen gesellschaftlichen Höherqualifizierung und der partiellen Modernisierung der Geschlechterrollen können wir im Vergleich zu früheren Milieustudien aber auch feststellen, dass insbesondere Männer, aber auch manche Frauen den Weg in regionale Fachhochschulen gefunden haben, wo sie u.a. technische und soziale Studiengänge wie Ingenieurwissenschaften und Soziale Arbeit belegen. Das Leben des traditionalen Milieus findet üblicherwei-

14 Vgl. dazu auch Illouz' (2009) Analyse der Kultur des Therapeutischen. 
se im dörflichen oder kleinstädtischen Kontext bzw. in der urbanen Siedlung statt, die Kontakte zu Verwandtschaft und Nachbarschaft sind eng. Paarbeziehungen folgen einer relativ traditionellen Arbeitsteilung.

Als Beispiel für die Deutungsweisen des traditionalen Milieus eignen sich die Trennungserzählungen von Jeanette und Sascha Schreiner. Die beiden kommen aus dem gleichen Ort und waren insgesamt zehn Jahre ein Paar. Zum Zeitpunkt des Kennenlernens waren sie in ihren Zwanzigern: Jeanette jobbte und sollte später eine Ausbildung zur Friseurin aufnehmen, der Bildungsaufsteiger Sascha studierte Ingenieurwesen an einer regionalen Fachhochschule. Nach drei Beziehungsjahren wurde Jeanette schwanger, was das Paar dazu veranlasste, gemeinsam in die Einliegerwohnung in Jeanettes Elternhaus zu ziehen und zu heiraten. Bald danach beendete Sascha sein Studium, wurde zum Familienernährer und übernahm im beruflichen Kontext zunehmend Verantwortung, derweil Jeanette beruflich pausierte und sich Wohnung und Kind widmete. In dieser Phase begann die Beziehung konfliktreich zu werden: Jeanette beschwerte sich über Konflikte mit ihren Eltern und fühlte sich von Sascha alleine gelassen. Beide berichteten auch von Konflikten um Geld. Im neunten Beziehungsjahr traf Jeanette dann ihre Jugendliebe Thorsten wieder und beichtete schließlich Sascha, sich verliebt zu haben. Sascha zog sich infolgedessen zurück und übernachtete auf dem Sofa, während sich Jeanette nun regelmäßig mit Thorsten traf. Die Trennung wurde schließlich von Jeanettes Eltern vorangetrieben, die Sascha baten zu handeln.

Aus Jeanettes Sicht liegt das Beziehungsscheitern darin begründet, dass Sascha nicht seinen Mann stand. Im Konflikt mit ihren Eltern habe er sich nicht auf ihre Seite gestellt, wie es ein guter Mann tun würde: „Ein Mann hat hinter seiner Frau zu stehen und net zwischendrin“ (JS 2016). Die finanziellen Probleme des Paares führt sie darauf zurück, dass er der „Spielsucht“ verfallen sei, während die ihr unterstellte „Kaufsucht“ nicht zutreffe: Bei ihren Bestellungen habe es sich zu ,90 Prozent“ um „Kinderklamotten und Kinderspielzeug“ gehandelt. Sie selbst als Frau mit bestimmten Konsumbedürfnissen habe sich dabei weit zurückgestellt und sei „fast bisschen vergammelt“. Ferner stellt sie ihr Fremdgehen als unausweichliche Konsequenz von Saschas Versagen als Mann dar. Zum einem habe er sich schon seit einiger Zeit sexuell zurückgezogen und nicht einmal mehr die Präsentation neuer Dessous zu würdigen gewusst. Zum anderen habe er nicht um sie gekämpft und ihr keine klaren Grenzen gesetzt, als sie ihn mit ihrer neuen Liebe konfrontierte. Damit verpasste er aus ihrer Sicht eine weitere Möglichkeit, sich als guter Ehemann zu erweisen:

JS: Ich bin jetzt nicht parallel fremdgegangen und hab ihm was vorgeheuchelt, von wegen ,ich lieb dich über alles und will mit dir zusammenbleiben“, sondern ich hab ihm von Anfang an ähm die Wahrheit eingeschenkt und er ist jetzt nicht wie jeder andere Mann, was normal gewesen wäre, mit der Faust auf den Tisch, ,ich trenn mich sofort und pack meine Sachen“ und weg ist er. Das hätte jeder andere Mann gemacht. Er aber nicht. Er hat das geschluckt, hat's in sich reingefressen, wobei ich sagen muss, er hat mich zu dem Zeitpunkt schon nicht mehr geliebt, weil jeder normale Mensch, wo mich lieben würd, würd sagen „und bis hierher und net weiter, äh Fräulein, du hast mich betrogen und tschüss“ 
und das hätt ich auch verdient. Ich hätt auch einen klaren Schnitt gebraucht zu dem Zeitpunkt. (JS 2016) ${ }^{15}$

Jeanette sieht also nicht ihre Parallelbeziehung als zentrales Problem, sondern Saschas ,unmännliche“ Schwäche, wobei sie im Nachhinein anerkennt, dass es dennoch „falsch gewesen“ sei, dass sie weiter mit Thorsten ausging. Gleichwohl dient ihr neues Beziehungsglück mit Thorsten als Bestätigung, dass es bessere Männer gibt.

Saschas Erzählung kann als komplettes Gegennarrativ zu Jeanettes Darstellung gelesen werden: Bezüglich einiger Ereignisse besteht zwar Einigkeit, nicht aber hinsichtlich ihrer Verursachung und Bedeutung. So habe Jeanette zum Beispiel ihre neue Beziehung, die sich nun nicht einmal als stabil erweise, verheimlicht. Generell rückt Sascha seine eigene zuverlässige Performanz als Mann, gerade als Ernährer, in den Vordergrund und stellt demgegenüber Jeanettes Performanz als Hausfrau und Mutter infrage. Indem sie ihn in die Konflikte mit ihren Eltern hineinzog, habe sie ihm nicht den Rücken freigehalten und ihm auf diese Weise den wohlverdienten entspannten Feierabend zu Hause verunmöglicht: „Ich komm heim von der Arbeit, hab ganz andere Probleme und dann muss ich mir das noch anhören“ (SaS 2016). Darüber hinaus wirft er ihr vor, dass sie das Familieneinkommen nicht gut verwalte, sondern insofern schmälere, als sie nach der Erziehungszeit des Kindes ,in so einen faulen Trott" verfallen sei, keinen Nebenjob mehr aufgenommen habe und stattdessen einer „Bestellsucht“ und „Kaufsucht“ verfallen sei, was die Geldprobleme verursacht habe. Außerdem negiert Sascha Jeanettes Leistung als Hausfrau: Auf einer Skala von 1 bis 10 schätzt er ihre diesbezüglichen Fähigkeiten lediglich als mittelmäßig (5-6) ein und kritisiert in diesem Zuge nicht nur ihre einseitige und für ihn ungenießbare Küche:

SaS: Was sie halt nicht so gemacht hat, gekocht. Sie hat dem Nico [dem Sohn, JE] oft nur Backofenpommes gemacht. Der wollt auch immer nur Pommes, aber da hat sie gemeint, wenn sie was anderes kocht, dann isst er es nicht und da hab ich gesagt, dann soll sie mal mit ihm zusammen kochen, dass er auch was klein schneiden darf und auch umrühren und dann will er es vielleicht auch mal essen und probieren und nicht nur immer was servieren. Oder für mich hat sie auch nicht viel gekocht. Oft hat sie mal so vegetarische Phasen gehabt, wo sie auf Fleisch verzichtet hat und hat dann im Prinzip nur für sich abends was Vegetarisches gekocht und für mich nix dann. (SaS 2016)

Dass Jeanette auch für das gemeinsame Kind nicht richtig kochte und seine ernährungspädagogischen Ideen nicht in die Tat umsetzte, wirft aus Saschas Sicht ferner ein negatives Licht auf Jeanettes Ausfüllung der Mutterrolle. Entsprechend gibt er sich bezüglich der Trennung nicht allzu traurig. Jeanettes Rat, in seiner neuen Beziehung mehr hinter der Frau zu stehen, stimmt ihn zwar leicht nachdenklich, aber rückwirkend kann er keinen Fehler erkennen: „Ich war oft nicht ihrer [Jeanettes, JE]

15 Für Goffman (1952, S. 457) stellt das Anbieten einer weiteren Chance, sich für die infrage stehende Rolle zu qualifizieren, eine mögliche Abkühlungsstrategie dar. Dazu vermerkt er allerdings auch, dass zweite Chancen selten ergriffen und dritte und vierte Chancen kaum gewährt würden. 
Meinung, sondern oft der Meinung der Schwiegereltern."Wie Jeanette konstruiert er also eine weitreichende Konstanz in der eigenen Identität: So wie sie für sich in Anspruch nimmt, durchweg ihre weibliche Rolle gut ausgefüllt zu haben, reklamiert er für sich eine gute Performanz in seiner männlichen Rolle. Dass die Beziehung scheiterte, liegt beiden zufolge vor allem in der mangelhaften Geschlechtsrollenperformanz der jeweils anderen Person begründet.

Von diesen veranschaulichenden Falleinblicken ausgehend kann für das traditionale Milieu somit festgehalten werden, dass Beziehungen als Geschlechterbeziehungen konzipiert sind, deren Gelingen in der adäquaten Geschlechtsrollenperformanz beider Partner*innen gesehen wird. Ein Authentizitätsanspruch wie im individualisierten Milieu wird dabei nicht erhoben: Rollen sind etwas, in das man hineinschlüpft und das man ausfüllt - nicht etwas, das man der individuellen Persönlichkeit und den eigenen Gefühlen entsprechend entwirft, gestaltet und auf authentische Weise verkörpert. Mit diesem Fokus auf die Geschlechtsrollenperformanz wird im traditionalen Milieu auch ein anderer Wirklichkeitsbegriff als im individualisierten Milieu verfolgt. Es geht nicht um eine innere Wahrheit, die tief im Subjekt verborgen liegt, sondern um eine gewissermaßen äußere Wahrheit, die problemlos allen Beobachter*innen des Geschehens zugänglich ist. Die Trennung wird als Konsequenz der auch für das soziale Umfeld offenkundig unzureichenden Geschlechtsrollenperformanz der anderen Person interpretiert, die als Mann oder als Frau versagte. Entsprechend dieser dezidierten Schuldzuweisung an die andere Person, die mit umfassendem Beweismaterial unterfüttert wird, dient das Interview als Anklagebank. Das eigene Selbst wird dabei maximal entlastet, man hat sich schließlich nichts vorzuwerfen und kann keine eigenen Fehler entdecken. Für sich selbst erheben die Interviewten den Identitätsanspruch als Mann oder Frau, der oder die konstant eine positive Rollenperformanz an den Tag legte - ein Anspruch, der angesichts des Gegennarrativs, das die Ex-Partner*in entwickelt und verbreitet, umso vehementer verteidigt wird.

\section{Milieuspezifische Deutungsrahmen und ihre Folgen für das Cooling- out}

Wie gezeigt existieren milieuspezifisch vorgefertigte Deutungsrahmen für die Redefinition des Trennungsgeschehens und die Wiederaufrichtung einer durch die Trennung beschädigten Identität, die von den Individuen ausgewählt und an ihre jeweilige Situation angepasst werden. Zur Analyse dieses Sachverhalts lässt sich das Coolingout-Konzept gewinnbringend mit Goffmans (1980 [1974]) Rahmenanalyse verbinden.

„Rahmen“ (,frames“) sind Goffman zufolge Interpretationsschemata, die „für das Verstehen von Ereignissen zur Verfügung stehen“ (ebd., S. 18) und auf diese Weise Alltagserfahrungen organisieren und kategorisieren helfen. Genauer gesagt liegen einerseits vorgefertigte, typische Rahmen vor, die sich durch ihre „Objektivität, Autonomie und Immunität gegenüber der faktischen (Inter-)Aktion auszeichnen“ (Willems 1996, S. 444) und entsprechend relativ stabil sind. Andererseits müssen die Rahmen als Sinnangebote in konkreten Situationen angewandt und aktualisiert 
werden, wobei diese „Rahmung“ (,framing“) für die Akteure aufgrund der Offenheit und Mehrdeutigkeit von Situationen durchaus herausfordernd ist (ebd.). Für Trennungen gilt das umso mehr, als es sich dabei nicht um eine spezifische, klar abgegrenzte Situation handelt, sondern um das Ergebnis einer ganzen Reihe von Ereignissen, Entscheidungen und Geschehnissen. Darüber hinaus ist ein kompletter Rahmenwechsel erforderlich: Eine, wie im Fall Chris Schmahl zu sehen, bis dahin noch gute oder zumindest funktionierende Beziehung entpuppt sich als gescheitert, was ein nachträgliches ,reframing“ der gesamten Beziehungsgeschichte nötig macht, die nun als Scheiternsgeschichte neu erzählt werden muss (Hopper 2001).

Die Verbindung von Cooling-out-Konzept und Rahmenanalyse ist auch insofern sinnvoll, als sich im Cooling-out-Aufsatz nur ein kurzer und zudem sehr vager Hinweis auf mögliche soziale Unterschiede bei der Deutung von Verlusten findet: „It should be noted, parenthetically“, so Goffman dort, ,that one circle of persons may define a particular loss as the kind that casts no reflection on the loser, and that a different circle of persons may treat the same loss as a symbol of what the loser deserves“ (1952, S. 454). In der Rahmenanalyse gibt es demgegenüber dezidiertere Hinweise auf die kulturelle Spezifizität der ,,primären Rahmen einer sozialen Gruppe“, die laut Goffman ,[z]usammengenommen [...] einen Hauptbestandteil von deren Kultur [bilden]“" (1980 [1974], S. 37). Je nach sozialem Kontext kann ein- und dieselbe Situationsrahmung also entweder als passend oder als sinnlos erscheinen. Bezüglich der hier interessierenden Trennungen können davon ausgehend Milieus als relevante „soziale Gruppe[n]“ (ebd.) gefasst werden. Wie anhand der Interviews von Getrennten aus dem individualisierten und dem traditionalen Milieu gezeigt, unterscheiden sich diese sozialen Gruppen in ihren Rahmenpräferenzen stark. So ist der individualisierte Rahmen bestimmt durch das Primat der Authentizität, die Suche nach einer verborgenen, inneren Wahrheit, die Relevanzhochstufung von Emotionen und die reflexive Arbeit am Selbst. Im traditionalen Rahmen hingegen dominieren die Zentralität der Geschlechtsrollenperformanz, die Annahme einer für alle sichtbaren, äußeren Wahrheit und die verlässliche Konstanz des Selbst in seiner Geschlechtsrolle.

Was aber folgt aus der Milieuspezifizität von Deutungsrahmen? Grundlegend ist festzuhalten, dass nicht jeder verfügbare Deutungsrahmen für jede*n ein adäquates Angebot darstellt. Individualisierte Interviewpartner*innen dürften traditionale Selbst- und Weltbilder genauso wenig anschlussfähig für das eigene Leben finden wie traditionale Interviewpartner*innen die Deutungen des individualisierten Milieus. Darüber hinaus ist anzunehmen, dass die „Wahl“ eines spezifischen Deutungsrahmens über ihre ordnungsstiftende und identitätssichernde Funktion hinaus weitere Konsequenzen für die Trennungsbewältigung hat, da Rahmen handlungsrelevant sind.

Für das individualisierte Milieu können diesbezüglich folgende Überlegungen angestellt werden: Die Suche nach einer ,inneren Wahrheit“ stellt sich als aufwendiger Prozess dar, bei dem konkrete Beobachtungen, Erlebnisse und Konflikte aus dem Paaralltag auf ihre tieferliegenden Ursachen zurückgeführt werden sollen. Dieses Verständnis hat Folgen dafür, wer als „,cooler“ infrage kommt und was deren spezifische Aufgabe ist. Zwar wird die innere Wahrheit in den Personen und ihren Biographien verortet, einem Territorium also, zu dem andere keinen Zugang haben. 
Jedoch können andere auf Basis der verfügbaren Informationen Interpretationsangebote unterbreiten oder eigene Interpretationen anregen und abstützen. Wird externe Hilfe bei der Trennungsbewältigung benötigt, erscheinen professionelle Persönlichkeitsdeuter*innen und Expert*innen für die zwischenmenschliche Kommunikation wie Psychotherapeut*innen - die Goffman (1952, S. 461) als ,the society's cooler“ bezeichnet - eine gute Adresse.

Der individualisierte Deutungsrahmen hat Vor- und Nachteile. Ein großer Vorteil einer aufgearbeiteten Trennung liegt in der Positivdeutung der gescheiterten Beziehung als schmerzhafte, aber gerade auch dadurch wichtige Erfahrung im persönlichen Reflexions- und Entwicklungsprozess. Schließlich kann jede Erfahrung im Narrativ der persönlichen Selbstfindung und Weiterentwicklung als sinnhaft umgedeutet werden. Des Weiteren dürfte die im individualisierten Milieu verbreitete Annahme, dass man selbst gewisse Handlungsspielräume hatte, aber die Beziehung aufgrund der Nicht-Passung der beiden Partner*innen scheiterte, die Anpassung an die neue Lebenssituation erleichtern. Ein möglicher Nachteil des individualisierten Deutungsrahmens liegt in der Komplexität der Deutungsaufgabe begründet: Die Suche nach und Analyse eines vermuteten zugrunde liegenden „Rieseneisbergs“ (CE 2017) kann in einen infiniten Regress münden, in dessen Zuge immer mehr offene Fragen und Persönlichkeitsbaustellen entdeckt werden. Solche ,,stärkere[n] Tendenzen des Nachdenkens und Grübelns über das Trennungsereignis“ könnten erklären, warum höher Gebildete stärker unter Trennungen leiden (Kopp et al. 2010, S. 178).

Im traditionalen Milieu gestaltet sich die Lage anders. Da die eigene Geschlechtsrollenperformanz sowie diejenige der Ex-Partner*in im Fokus stehen - und damit Handlungen, die für Beobachter*innen unmittelbar sichtbar sind -, stellen Augenzeug*innen aus dem unmittelbaren sozialräumlichen Umfeld die primären „cooler“ dar. Ihre Aufgabe besteht darin, die eigene Normkonformität sowie den Normbruch der anderen Person zu bestätigen - auch durch das Einbringen weiteren belastenden „Materials“. Gute Zeug*innen können gemeinsame Bekannte oder Freund*innen des Paares sein, wie sie Jeanette Schreiner in den Zeugenstand ruft, um ihre These von Saschas Wandel zu bestätigen. Ferner zitiert sie ihre Freundinnen, um Saschas Männlichkeitsdefizit zu belegen: „Meine Freundinnen haben immer gesagt, der hat keine Eier in der Hose, hat jede gesagt“. Doch auch Sascha kann auf Zeug*innen für Jeanettes mangelnde Rollenperformanz verweisen: Die auf Jeanettes „Kaufsucht“ zurückgehenden regelmäßigen Paketlieferungen seien ,natürlich den ganzen Nachbarn aufgefallen und auch den Schwiegereltern, die haben mich schon darauf angesprochen“. Therapeut*innen hingegen stellen keine relevanten „cooler“ dar: Sie können nicht mehr sehen oder besser beurteilen als die Beteiligten selbst. Nur sporadisch wird auf Autoritäten und Repräsentant*innen der gesellschaftlichen Ordnung verwiesen, die durch die Kenntnis der „objektiven“ Ereignisse und Handlungsweisen gemäß der herrschenden Moral urteilen. Jeanette Schreiner beispielsweise beruft sich auf Erzieherinnen im Kindergarten ihres Sohnes, um sich die Angemessenheit ihres Erziehungsstils von Fachkundigen bescheinigen zu lassen und so ihre gute Ausfüllung der Mutterrolle zu untermauern.

Gelingt es Angehörigen des traditionalen Milieus, eine hinreichende Menge an Zeug*innen zu mobilisieren, die die eigene Version der Geschichte bekräftigen, dann sollte die Trennung gut bewältigt werden können, da das Selbst nicht grundlegend 
infrage gestellt wird. Förderlich ist es auch, wenn eine neue Beziehung zu einer Person eingegangen wird, die einer sozial geteilten Wahrnehmung zufolge eine bessere Geschlechterrollenperformanz an den Tag legt als die Ex-Partner*in. Dies gilt als Beleg für die Angemessenheit der eigenen Erwartungshaltung, die offenbar erfüllbar ist und daher nicht überzogen war, sowie für die unzureichende Rollenperformanz der Ex-Partner*in. Darüber hinaus gilt eine neue Paarbeziehung als Hinweis auf die eigene gute Rollenperformanz, die von der neuen Partner*in durch das Eingehen der Beziehung bestätigt wird. Erschwert wird der Cooling-out-Prozess hingegen, wenn Nachbar*innen, Freund*innen, Familie und Verwandtschaft, zu denen im traditionalen Milieu üblicherweise ein regelmäßiger Kontakt besteht, Kritik am eigenen Handeln äußern. Dies dürfte für Jeanette zutreffen, deren Eltern den Diplomingenieur Sascha für eine außerordentlich gute Partie und - laut Sascha - Jeanettes Rollenperformanz für unzureichend hielten, zumindest was das „Rückenfreihalten“ angeht: „Das haben auch meine Eltern oder Schwiegereltern gesagt, das hat sie nicht so gut gemacht“. Als problematisch für den Cooling-out-Prozess speziell der Verlassenen kann sich darüber hinaus auswirken, dass aufgrund der alleinigen Schuldzuweisung an die Ex-Partner*in keine eigenen Handlungsspielräume und damit Kontrollmöglichkeiten gesehen werden (Amato und Previti 2003, S. 620; Gray und Silver 1990, S. 1189; Grych und Fincham 1992; Kopp et al. 2010, S. 183).

\section{Schluss}

In diesem Beitrag habe ich ein doppeltes Anliegen verfolgt. Zum einen habe ich die familiensoziologische Relevanz des Cooling-out-Konzepts mit Blick auf das Beziehungsscheitern herausgearbeitet, das auch in Zeiten seiner Normalisierung sowie öffentlicher Entproblematisierung und Entstigmatisierung eine Identitätsbedrohung darstellt. Relevant ist das Cooling-out-Konzept hierbei als integrative soziologische Konzeptualisierung, die es erlaubt, neben den ökonomischen Trennungsverlusten auch und gerade die verschiedenen trennungsinduzierten interpretativen Sinn-, Selbst- und Planungskrisen in den Blick zu nehmen. Darüber hinaus verweist Goffmans Konzept darauf, dass eine erfolgreiche Krisenbewältigung die situationsbedingte Neudeutung des ,Selbst“ sowie soziale Unterstützung in dieser Hinsicht erfordert. Goffmans soziologische Konzeptualisierung weist dabei über die in der familiensoziologischen Forschung bisher überwiegende Analyse interpretativer Krisen mittels psychologischer Konzepte hinaus, denn sie erlaubt, den Blick auf das soziale Umfeld und die soziale Position der Betroffenen, hier hinsichtlich ihrer Milieuzugehörigkeit, zu lenken.

Zum anderen habe ich empirische Erkenntnisse aus der Trennungsforschung für eine Weiterentwicklung des Cooling-out-Konzepts genutzt. Deutlich wurde erstens, dass Cooling-out zumindest bei Trennungen keine einmalige Situation darstellt, sondern als langwieriger Prozess zu betrachten ist. Zweitens gibt es auch Konstellationen mit zwei Verlierer*innen und mindestens zwei „,coolers“, was Goffmans klare Rollentrennung von „,mark“ und „,cooler“ verkompliziert. Drittens werden Deutungen für die Redefinition der Situation und des Selbst nicht spontan und idiosynkratisch erschaffen. Vielmehr konnten in den Interviews milieuspezifische Deutungs- 
rahmen identifiziert werden, was auf soziale Unterschiede im Umgang mit dem Beziehungsscheitern verweist und eine Weiterführung des Cooling-out-Konzepts mithilfe von Goffmans Rahmenanalyse nahelegt.

So haben ,cooler“ im individualisierten Milieu die Aufgabe, die Ex-Partner*in bei der (Re-)Konstruktion der inneren, verborgenen Wahrheit und damit der tatsächlich oder vermeintlich der Trennung zugrundeliegenden Persönlichkeits- und Beziehungsmechanismen zu unterstützen. Neben Personen aus dem eigenen sozialen Umfeld sind hierfür insbesondere Psychotherapeut*innen relevant. Von Vorteil ist diese Herangehensweise, wenn die Trennungserfahrung letztlich als wertvoller Beitrag zum persönlichen Reflexions- und Entwicklungsprozess evaluiert werden kann. Von Nachteil ist sie mutmaßlich, wenn im Zuge eines nicht enden wollenden Reflexionsprozesses das Selbst nachhaltig hinterfragt wird. Im traditionalen Milieu sieht es gänzlich anders aus: Präferierte „,cooler“ sind hier Augenzeug*innen aus dem unmittelbaren sozialen Umfeld, die den Normbruch der anderen Person und die Normkonformität des eigenen Handelns im Hinblick auf die jeweiligen Geschlechterrollen bestätigen. Bewältigungsförderlich ist es, wenn eine solche Zeugenschaft mobilisiert werden kann und das Selbst dadurch nicht grundlegend infrage gestellt wird. Allerdings erscheint es schwerer, einer gescheiterten Beziehung per se etwas Positives abzugewinnen und ihr damit einen Sinn zu verleihen - es sei denn, die Trennung ermöglicht das Eingehen einer neuen Beziehung mit einer rollenkonform agierenden Person.

Insgesamt ergibt diese Perspektive, dass sich die Konstruktion neuer Situationsund Selbstdefinitionen im Wechselspiel mentaler und interaktiver Prozesse sowie kultureller und milieuspezifischer Deutungsmuster vollzieht. Interpretation um Interpretation, Interaktion um Interaktion werden Trennungsdeutungen und Identitäten entworfen und interaktiv abgesichert (vgl. Malone 1997), ohne jemals gänzlich fixiert zu sein.

Auf Basis dessen lassen sich weitere Forschungsmöglichkeiten skizzieren. So kann gefragt werden, unter welchen Bedingungen das Cooling-out misslingt, etwa weil die soziale Unterstützung fehlt, weil keine passenden milieuspezifischen Deutungsrahmen verfügbar sind oder weil das Scheitern als so umfänglich erlebt wird, dass eine positive Neudeutung kaum möglich ist. In unserem empirischen Material scheint letzteres für eine der vier von uns identifizierten Scheiternskonstellationen, nämlich die gescheiterte Status(re)produktion, besonders relevant zu sein: Eine Person trennt sich, als die andere in einer anhaltenden beruflichen Krise steckt, was aus Sicht der sich trennenden Person die von ihr angestrebte Status(re)produktion im Paarzusammenhang verunmöglicht (Eckert et al. 2021, S. 269 ff.). ${ }^{16}$ Die Verlassenen sind dadurch gleich mit zwei Problemen konfrontiert: dem beruflichen Scheitern und dem Beziehungsscheitern. Damit verbunden sind zwei Rollentode, was an die von Goffman (1952, S. 461) beschriebene Totalerschütterung einer Person erinnert.

\footnotetext{
16 Daneben ließen sich drei weitere Konstellationen und Dynamiken rekonstruieren, die das Beziehungsscheitern erklären (im Überblick: Eckert et al. 2021): fortwährende und große Machtungleichgewichte symbolischer und emotionaler Art zwischen den Partner*innen (ausführlich dazu Koppetsch et al. 2019), anhaltend einseitige Fürsorgearrangements sowie die Desynchronisierung der wechselseitigen - auch geschlechtlich codierten - Erwartungen im Lebensverlauf.
} 
Im Vergleich zu den Verlassenen anderer Scheiternskonstellationen erscheinen die Folgen für diesen Typus von Getrennten besonders gravierend. Nicht nur kurz-, sondern auch mittel- und teils gar langfristig kommen sie nur schwer über die erfahrenen Kränkungen hinweg. Sie werden depressiv, erleiden Zusammenbrüche und teils einen weiteren beruflichen Abstieg, was es vermutlich äußerst schwierig werden lässt, sich selbst in einem neuen, positiven Licht zu sehen.

Darüber hinaus könnte die These, dass Deutungsrahmen und die Verarbeitung einer Trennungserfahrung milieuspezifisch organisiert sind, weitere Untersuchungen anregen, etwa hinsichtlich der Deutungsrahmen weiterer akademischer und nichtakademischer Milieus. Anders als in der vorliegenden Studie sollten dabei auch ostdeutsche Interviewpartner*innen einbezogen werden, da in familien- und geschlechtersoziologischen Studien auch noch Jahrzehnte nach der Wende eine ,erstaunliche Persistenz kultureller Unterschiede zwischen Ostdeutschen und Westdeutschen“ (Behnke 2012, S. 115) festgestellt wurde, was auf unterschiedliche Sozialisationserfahrungen in der DDR und BRD zurückgeführt wird. Dies wirft zugleich die Frage nach der Relevanz aktueller landesspezifischer Kontextbedingungen auf: Ähneln sich individualisiert orientierte alternative Akademiker*innen sowie traditional orientierte Arbeiter*innen in ihren Deutungsmustern hierzulande und in anderen westlichen Gesellschaften, wie manche Autor*innen nahelegen (Illouz 2009)? Oder gibt es hier einen großen Differenzierungsbedarf, wie andere betonen (Lamont 2000)?

Antworten auf diese Fragen könnten nicht nur für Soziolog*innen, sondern auch für Praktiker*innen von Therapeut*innen über Sozialarbeiter*innen hin zu Familienrichter*innen relevant sein, die mit Getrennten arbeiten, ihre Version der Geschichte einordnen und ihnen eine adäquate Unterstützung oder Behandlung zukommen lassen wollen.

Funding Open Access funding enabled and organized by Projekt DEAL.

Open Access Dieser Artikel wird unter der Creative Commons Namensnennung 4.0 International Lizenz veröffentlicht, welche die Nutzung, Vervielfältigung, Bearbeitung, Verbreitung und Wiedergabe in jeglichem Medium und Format erlaubt, sofern Sie den/die ursprünglichen Autor(en) und die Quelle ordnungsgemäß nennen, einen Link zur Creative Commons Lizenz beifügen und angeben, ob Änderungen vorgenommen wurden.

Die in diesem Artikel enthaltenen Bilder und sonstiges Drittmaterial unterliegen ebenfalls der genannten Creative Commons Lizenz, sofern sich aus der Abbildungslegende nichts anderes ergibt. Sofern das betreffende Material nicht unter der genannten Creative Commons Lizenz steht und die betreffende Handlung nicht nach gesetzlichen Vorschriften erlaubt ist, ist für die oben aufgeführten Weiterverwendungen des Materials die Einwilligung des jeweiligen Rechteinhabers einzuholen.

Weitere Details zur Lizenz entnehmen Sie bitte der Lizenzinformation auf http://creativecommons.org/ licenses/by/4.0/deed.de.

\section{Literatur}

\section{Verwendete Literatur}

Amato, P. R. (2000). The consequences of divorce for adults and children. Journal of Marriage and Family, $62,1269-1287$. 
Amato, P. R. (2014). The consequences of divorce for adults and children: An update. Društvena Istraživanja, 23, 5-24.

Amato, P. R., \& Previti, D. (2003). People's reasons for divorcing. Gender, social class, the life course, and adjustment. Journal of Family Issues, 24, 602-626.

Andreß, H.-J. (2003). Wenn aus Liebe rote Zahlen werden. Über die wirtschaftlichen Folgen von Trennung und Scheidung. Wiesbaden: Westdeutscher Verlag.

Arosio, L. (2016). Old and new rites of passage in contemporary Western societies: A focus on marriage and divorce ceremonies. Journal of Comparative Research in Anthropology and Sociology, 7, 91-104.

Arránz Becker, O. (2015). Determinanten und Konsequenzen von Trennung und Scheidung. In P. B. Hill \& J. Kopp (Hrsg.), Handbuch Familiensoziologie (S. 527-561). Wiesbaden: Springer VS.

Backert, W. (2004). Kulturen des Scheiterns: Gesellschaftliche Bewertungsprozesse im internationalen Vergleich. In M. Junge \& G. Lechner (Hrsg.), Scheitern. Aspekte eines sozialen Phänomens (S. 63-77). Wiesbaden: VS Verlag für Sozialwissenschaften.

Beelmann, W. (1994). Stressbelastung und Stressbewältigung bei der Auseinandersetzung mit einer Trennung vom Ehepartner. Eine empirische Langzeitstudie. Regensburg: S. Roderer.

Behnke, C. (2012). Partnerschaftliche Arrangements und väterliche Praxis in Ost- und Westdeutschland. Paare erzählen. Opladen u.a.: Barbara Budrich.

Berger, P. L., \& Kellner, H. (1965). Die Ehe und die Konstruktion der Wirklichkeit: Eine Abhandlung zur Mikrosoziologie des Wissens. Soziale Welt, 16, 220-235.

Bourdieu, P. (1982). Die feinen Unterschiede. Kritik der gesellschaftlichen Urteilskraft. Frankfurt a. M.: Suhrkamp.

Bröckel, M., \& Andreß, H.-J. (2015). The economic consequences of divorce in Germany: What has changed since the turn of the millennium? Comparative Population Studies, 40, 277-312.

Clark, B. R. (1960). The „cooling out“ function in higher education. American Journal of Sociology, 65, 569-576.

Doering, J. (2010). Face, accounts, and schemes in the context of relationship breakups. Symbolic Interaction, 33, 71-95.

Eckert, J., Bub, E.-M., \& Koppetsch, C. (2021). Warum scheitern Beziehungen? Theoretisch-konzeptionelle Überlegungen und empirische Ergebnisse. In M. Wutzler, \& J. Klesse (Hrsg.), Paarbeziehungen heute: Kontinuität und Wandel (S. 249-279). Weinheim \& Basel: Beltz Juventa.

Eckert, J., Bub, E.-M., \& Koppetsch, C. (2019). Über Trennungen erzählen: zur Milieuspezifik von Trennungslegitimationen und narrativen Identitäten. Forum Qualitative Sozialforschung / Forum: Qualitative Social Research, 20. URL: https:/www.qualitative-research.net/index.php/fqs/article/view/3078/ 4351. Zugegriffen: Aug. 2021.

Engler, S. (2018). Annullieren statt akzeptieren. Kirchliche Ehenichtigkeitsverfahren. Herder Korrespondenz, 72, 44-46.

Filipp, S.-H., \& Aymanns, P. (2018). Kritische Lebensereignisse und Lebenskrisen. Vom Umgang mit den Schattenseiten des Lebens. 2., aktualisierte Aufl. Stuttgart: Kohlhammer.

Frevert, U. (2009). Was haben Gefühle in der Geschichte zu suchen? Geschichte und Gesellschaft, 35, $183-208$.

Gerstel, N. (1987). Divorce and stigma. Social Problems, 34, 172-186.

Goffman, E. (1952). On cooling the mark out. Some aspects of adaptation to failure. Journal for the Study of Interpersonal Processes, 15, 451-463.

Goffman, E. (1975 [1963]). Stigma. Über Techniken der Bewältigung beschädigter Identität. Frankfurt a. M.: Suhrkamp.

Goffman, E. (1980 [1974]). Rahmen-Analyse. Ein Versuch über die Organisation von Alltagserfahrungen. Frankfurt a. M.: Suhrkamp.

Gray, J. D., \& Silver, R. C. (1990). Opposite sides of the same coin. Former spouses' divergent perspectives in coping with their divorce. Journal of Personality and Social Psychology, 59, 1180-1191.

Grych, J. H., \& Fincham, F. D. (1992). Marital dissolution and family adjustment: An attributional analysis. In T. L. Orbuch (Hrsg.), Close relationship loss. Theoretical approaches (S. 157-173). New York: Springer.

Herzer, M. (2006). Ehescheidung als sozialer Prozess. Mainz: o.A. URL: https://d-nb.info/1119176069/ 34. Zugegriffen: Aug. 2020.

Hill, P. B., \& Kopp, J. (2015). Editorial on the special issue "Research on divorce: Causes and consequences". Comparative Population Studies, 40, 219-228.

Hopper, J. (2001). The symbolic origins of conflict in divorce. Journal of Marriage and Family, 63, $430-445$. 
Illouz, E. (2009). Die Errettung der modernen Seele. Therapien, Gefühle und die Kultur der Selbsthilfe. Frankfurt a. M.: Suhrkamp.

Kopp, J., Arránz Becker, O., Kunz, C., \& Lois, D. (2010). Verliebt, verlobt, verheiratet. Institutionalisierungsprozesse in Partnerschaften. Wiesbaden: VS Verlag für Sozialwissenschaften.

Koppetsch, C., \& Burkart, G. (1999). Die Illusion der Emanzipation. Zur Wirksamkeit latenter Geschlechtsnormen im Milieuvergleich. Konstanz: UVK.

Koppetsch, C., \& Speck, S. (2015). Wenn der Mann kein Ernährer mehr ist. Geschlechterkonflikte in Krisenzeiten. Berlin: Suhrkamp.

Koppetsch, C., Bub, E.-M., \& Eckert, J. (2019). Bindungsmacht in Paarbeziehungen. Warum Beziehungen scheitern. Berliner Journal für Soziologie, 29, 5-32.

Kruse, J. (2015). Qualitative Interviewforschung. Ein integrativer Ansatz. 2., überarbeitete und ergänzte Aufl. Weinheim \& Basel: Beltz Juventa.

Lamont, M. (2000). The dignity of working men. Morality and the boundaries of race, class, and immigration. Cambridge (USA) \& New York: Harvard University Press \& Russell Sage Foundation.

Lenz, K. (2006). Soziologie der Zweierbeziehung. Eine Einführung. 3. Aufl. Wiesbaden: VS Verlag für Sozialwissenschaften.

Lucius-Hoene, G., \& Deppermann, A. (2004). Rekonstruktion narrativer Identität. Ein Arbeitsbuch zur Analyse narrativer Interviews. 2. Aufl. Wiesbaden: VS Verlag für Sozialwissenschaften.

Malone, M. (1997). Worlds of talk: The presentation of self in everyday conversation. Cambridge (UK): Polity Press.

McCall, G. J. (1982). Becoming unrelated: The management of bond dissolution. In S. Duck (Hrsg.), Dissolving personal relationships (S. 211-231). New York: Academic.

Mühling, T., \& Schreyer, J. (2012): Beziehungsverläufe in West- und Ostdeutschland - Stabilität und Übergänge. ifb-Materialien 2012/4. URL: https://www.ifb.bayern.de/imperia/md/content/stmas/ifb/ materialien/mat_2012_4.pdf. Zugegriffen: Aug. 2020.

Nadai, E. (2007). Cooling out. Zur Aussortierung von „Leistungsschwachen“. In C. Arni (Hrsg.), Der Eigensinn des Materials: Erkundungen sozialer Wirklichkeit (S. 445-461). Frankfurt a. M. \& Basel: Stroemfeld.

Peuckert, R. (2019). Familienformen im sozialen Wandel. 9. Aufl. Wiesbaden: Springer VS.

Riessman, C. K. (1990). Divorce talk. Women and men make sense of personal relationships. New Brunswick: Rutgers University Press.

Rosenthal, G. (2014). Interpretative Sozialforschung. Eine Einführung. 4. Aufl. Weinheim \& Basel: Beltz Juventa.

Ruspini, E. (2013). Culture of divorce. In R. E. Emery (Hrsg.), Cultural sociology of divorce. An encyclopedia. Volume $1(A-F)$ (S. 336-341). Thousand Oaks: SAGE Reference.

Sagstetter, E.-M. (1989). Belastungssituationen und Bewältigungsformen emotionaler Probleme bei der Trennung und Scheidung vom Ehepartner. Dissertation. Regensburg: Universität Regensburg.

Schmidt, G., Matthiesen, S., Dekker, A., \& Starke, K. (2006). Spätmoderne Beziehungswelten. Report über Partnerschaft und Sexualität in drei Generationen. Wiesbaden: VS Verlag für Sozialwissenschaften.

Schneider, N. (1990). Woran scheitern Partnerschaften? Subjektive Trennungsgründe und Belastungsfaktoren bei Ehepaaren und nichtehelichen Lebensgemeinschaften. Zeitschrift für Soziologie, 19, 458-470.

Schöningh, I., Aslanidis, M., \& Faubel-Diekmann, S. (1991). Alleinerziehende Frauen. Zwischen Lebenskrise und neuem Selbstverständnis. Opladen: Leske + Budrich.

Singly, F. de (2011). Séparée. Vivre l'expérience de la rupture. Paris: Armand Colin.

Snow, D. A., Robinson, C., \& McCall, P. L. (1991). "Cooling out" men in single bars and nightclubs. Observations on the interpersonal survival strategies of women in public places. Journal of Contemporary Ethnography, 19, 423-449.

Statistisches Bundesamt (2018). Deutlich weniger Ehescheidungen im Jahr 2017. Pressemitteilung Nr. 251 vom 10. Juli 2018. URL: https://www.destatis.de/DE/Presse/Pressemitteilungen/2018/07/PD18_ 251_12631.html. Zugegriffen: Aug. 2020.

Strauss, A. L., \& Corbin, J. M. (1996). Grounded Theory: Grundlagen qualitativer Sozialforschung. Weinheim: Beltz Psychologie-Verlags-Union.

Thomas, G. M. (2014). Cooling the mother out: Revisiting and revising Goffman's account. Symbolic Interaction, 37, 283-299.

Usera, D. (2018). "Cooling the mark out" in relationship dissolution. Kentucky Journal of Communication, 37, 4-22.

Vaughan, D. (1988). Wenn Liebe keine Zukunft hat. Stationen und Strategien der Trennung. Reinbek: Rowohlt. 
Walzer, S., \& Oles, T. P. (2003). Accounting for divorce: Gender and uncoupling narratives. Qualitative Sociology, 26, 331-349.

Wang, H., \& Amato, P. R. (2000). Predictors of divorce adjustment: Stressors, resources, and definitions. Journal of Marriage and Family, 62, 655-668.

Wei, J. (2016). "I'm the next American idol": Cooling out, accounts, and perseverance at reality talent show auditions. Symbolic Interaction, 39, 3-25.

Weiss, R. S. (1980). Trennung vom Ehepartner. Stuttgart: Klett-Cotta.

Werneck, H. (2004). Auswirkungen einer Scheidung auf Befindlichkeit und Persönlichkeitsentwicklung. In U. Zartler, L. Wilk, \& R. Kränzl-Nagl (Hrsg.), Wenn Eltern sich trennen. Wie Kinder, Frauen und Männer Scheidung erleben (S. 247-279). Frankfurt a. M.: Campus.

Willems, H. (1996). Goffmans qualitative Sozialforschung. Ein Vergleich mit Konversationsanalyse und Strukturaler Hermeneutik. Zeitschrift für Soziologie, 25, 438-455.

Zartler, U. (2012). Die Kernfamilie als Ideal. Zur Konstruktion von Scheidung und Nachscheidungsfamilien. Zeitschrift für Familienforschung, 24, 67-84.

\section{Zitierte Interviews}

CE 2017: Teil-narratives Interview mit Charlotte Epp (Pseudonym), August 2017.

CS 2016: Teil-narratives Interview mit Chris Schmahl (Pseudonym), November 2016.

HS 2016: Teil-narratives Interview mit Helen Schmahl (Pseudonym), November 2016.

JS 2016: Teil-narratives Interview mit Jeanette Schreiner (Pseudonym), Dezember 2016.

KA 2017: Teil-narratives Interview mit Konstantin Auth (Pseudonym), Juni 2017.

NU 2017: Teil-narratives Interview mit Nora Uhlmann (Pseudonym), Oktober 2017.

SaS 2016: Teil-narratives Interview mit Sascha Schreiner (Pseudonym), Dezember 2016.

Judith Eckert geb. 1985, Dr. phil., Wissenschaftliche Mitarbeiterin im DFG-geförderten Projekt „Fragen in qualitativen Interviews. Sekundäranalysen zur Bedeutung unterschiedlicher Frageformen in Interviews" an der Universität Duisburg-Essen. Forschungsschwerpunkte: Qualitative Methoden, Kultur- und Wissenssoziologie, Soziologie der Angst, Beziehungs- und Familiensoziologie, Geschlechterforschung, soziale Ungleichheit. Ausgewählte aktuelle Veröffentlichungen: Gesellschaft in Angst? Zur theoretischempirischen Kritik einer populären Zeitdiagnose, 2019; (mit D. Cichecki) Mit „gescheiterten“ Interviews arbeiten. Impulse für eine reflexiv-interaktionistische Interviewforschung, 2020; (mit E.-M. Bub und C. Koppetsch) Warum scheitern Beziehungen? Theoretisch-konzeptionelle Überlegungen und empirische Ergebnisse, in M. Wutzler \& J. Klesse (Hrsg.), Paarbeziehungen heute: Kontinuität und Wandel, 2021. 